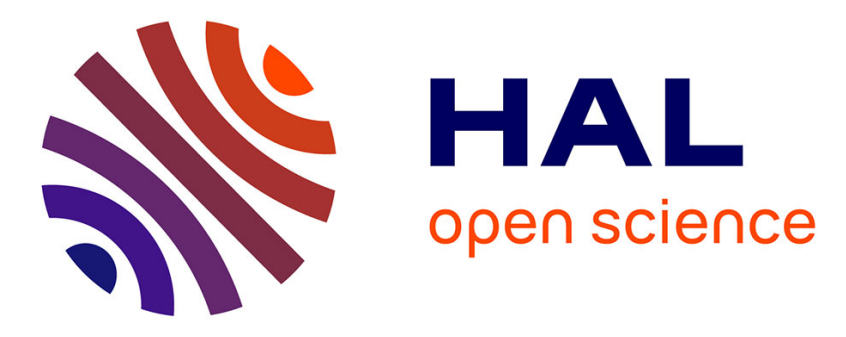

\title{
Acto-myosin network geometry defines centrosome position
}

Ana Joaquina Jimenez, Alexandre Schaeffer, Chiara de Pascalis, Gaëlle

Letort, Benoit Vianay, Michel Bornens, Matthieu Piel, Laurent Blanchoin,

Manuel Théry

\section{To cite this version:}

Ana Joaquina Jimenez, Alexandre Schaeffer, Chiara de Pascalis, Gaëlle Letort, Benoit Vianay, et al. Acto-myosin network geometry defines centrosome position. Current Biology - CB, 2021, 31 (6), pp.1206-1220.e5. 10.1016/j.cub.2021.01.002 . hal-03186011

\section{HAL Id: hal-03186011 \\ https://hal.science/hal-03186011}

Submitted on 31 Mar 2021

HAL is a multi-disciplinary open access archive for the deposit and dissemination of scientific research documents, whether they are published or not. The documents may come from teaching and research institutions in France or abroad, or from public or private research centers.
L'archive ouverte pluridisciplinaire HAL, est destinée au dépôt et à la diffusion de documents scientifiques de niveau recherche, publiés ou non, émanant des établissements d'enseignement et de recherche français ou étrangers, des laboratoires publics ou privés.

\section{()ㅜ) $\Theta$}

Distributed under a Creative Commons Attribution - NoDerivatives| 4.0 International 


\title{
Acto-myosin network geometry defines centrosome position.
}

\author{
Ana Joaquina Jimenez ${ }^{1}$, Alexandre Schaeffer ${ }^{1}$, Chiara De Pascalis ${ }^{1}$, Gaëlle Letort ${ }^{2}$, Benoit Vianay ${ }^{1}$, Michel \\ Bornens $^{3}$, Matthieu Piel ${ }^{3,4}$, Laurent Blanchoin ${ }^{1,5}$, Manuel Théry ${ }^{1,5}$ \\ 1- Univ. Paris, INSERM, CEA, UMRS1160, Institut de Recherche Saint Louis, CytoMorpho Lab, Hôpital Saint Louis, 75010 Paris, France. \\ 2- CIRB, Collège de France, UMR7241/U1050, Paris, France. \\ 3- Institut Curie, UMR144, Paris, France. \\ 4- Institut Pierre-Gilles de Gennes, Paris, France. \\ 5- Univ. Grenoble-Alpes, CEA, CNRS, INRA, Interdisciplinary Research Institute of Grenoble, Laboratoire de Phyiologie Cellulaire \& \\ Végétale, CytoMorpho Lab, 38054 Grenoble, France. \\ Correspondence should be addressed to: manuel.thery@cea.fr
}

The centrosome is the main organizer of microtubules and as such, its position is a key determinant of polarized cell functions. As the name says, the default position of the centrosome is considered to be the cell geometrical center. However, the mechanism regulating centrosome positioning is still unclear and often confused with the mechanism regulating the position of the nucleus to which it is linked. Here we used enucleated cells plated on adhesive micropatterns to impose regular and precise geometrical conditions to centrosome-microtubule networks. Although frequently observed there, the equilibrium position of the centrosome is not systematically at the cell geometrical center and can be close to cell edge. Centrosome positioning appears to respond accurately to the architecture and anisotropy of the actin network, which constitutes, rather than cell shape, the actual spatial boundary conditions the microtubule network is sensitive to. We found that the contraction of the actin network defines a peripheral margin, in which microtubules appear bent by compressive forces. The progressive disassembly of the actin network at distance from the cell edges defines an inner zone where actin bundles were absent, where microtubules were more radially organized and where dynein concentration was higher. We further showed that the production of dynein-based forces on microtubules places the centrosome at the center of this zone. In conclusion, the spatial distribution of cell adhesion and the production of contractile forces define the architecture of the actin network with respect to which the centrosome-microtubule network is centered. 


\section{Introduction}

The centrosome position is intimately associated to polarized cell functions such as adsorption and secretion, motility and mitosis (Tang and Marshall, 2012). Its position is characteristic and indicative of polarized cell functions (Bornens, 2018). It is found at the cell center in proliferating cells in culture, while it presents a peripheral position in differentiated cells in tissues, where it loses part or all of its functions in microtubule organization (Burakov and Nadezhdina, 2020; Sanchez and Feldman, 2016; Vallee and Stehman, 2005). During several cellular events essential to development, and organism homeostasis, the centrosome position undergoes a shift from the center to periphery of the cell, notably during ciliogenesis (Pitaval et al., 2017), neuronal developement (Shao et al., 2020), immune synapse formation (Stinchcombe and Griffiths, 2014) or epithelial-to-mesenchymal transition (Burute et al., 2017). However the mechanisms that regulate the stability of central and peripheral states and those that allow a rapid switch between two states have not yet been fully understood.

Previous in vivo, in vitro and in silico studies suggest that centrosome position is the outcome of a balance of pulling and pushing forces applied on microtubules and transmitted to the centrosome (Kimura and Kimura, 2011; Zhu et al., 2010). Overexpression or depletion of dynein heavy chains or its partners, and injections of dynein blocking antibodies suggest cortical and cytoplasmic dynein play a role in the production of pulling forces for the centrosome position (Burakov et al., 2003; Koonce, 1999; Nguyen-Ngoc et al., 2007; Tanimoto et al., 2016; Wu et al., 2011). Besides, microtubule polymerization against spatial boundaries have been shown to be responsible for the production of pushing forces (Brito et al., 2005; Garzon-Coral et al., 2016; Pinot et al., 2009). The exact role of actomyosin contration is unclear. The inhibition of actomyosin contraction had no visible effect on centrosome position in isolated cells or in monolayers (Hale et al., 2011), however it was found capable to counteract the centrosome shifting due to local microtubule disassembly (Burakov et al., 2003) and to perturb centrosome repositioning at the cell center after mitosis (Chevrier, 2002).

In non-differentiated cells, and notably in cells proliferating in culture, the force balance is believed to set the centrosome position at the cell geometrical center, also called center of mass or centroid (Graham et al., 2018; Hale et al., 2011; Théry et al., 2006a; Vallee and Stehman, 2005). Microtubule-based forces in an in vitro reconstituted system also position the MTOC at the centroid of their confinement area (Laan et al., 2012) but in silico simulation suggested this mechanism may depend on the shape and physical properties of the boundaries (Letort et al., 2016; Pavin et al., 2012). Indeed, in cultured cells, the centrosome has been observed at the cell geometrical center in relatively isotropic boundary conditions (e.g. non polarized cells) but can be off-centered in the front or in the back of migrating cells (Luxton and Gundersen, 2011; Pouthas et al., 2008; Zhang and Wang, 2017), or toward intercellular junction in epithelial cells (Burute et al., 2017; Rodriguez-Fraticelli et al., 2012). As a result, there is no generic definition of the centrosome position and the key parameters involved in the regulation of this positioning are still unclear. 
One limitation for the identification of the forces exerted on the centrosome is that the mechanism of centrosome positioning is hardly distinguishable from nucleus positioning. It has been a considerable limitation for the study of centrosome positioning in anisotropic conditions such as in migrating cells (Dupin et al., 2009; Gomes et al., 2005). Both the nucleus and the centrosome have their own self-centering properties (Dupin and EtienneManneville, 2011; Kimura and Kimura, 2011; Reinsch and Gönczy, 1998). But the physical links that connect them hinder their respective contributions in regards to their final position (Bornens, 1977; Burakov and Nadezhdina, 2013; Malone et al., 2003; Salpingidou et al., 2007; Zhang et al., 2009). In addition, the nucleus also constitutes a dead volume microtubules don't have access to, which biases the spatial distribution of microtubules and their associated forces (Luxton and Gundersen, 2011). Furthermore, centrosomal microtubules push and pull on the nuclear envelop (Biedzinski et al., 2020; Burke and Roux, 2009; Starr and Fridolfsson, 2010) adding more complexity to the force balance in the centrosome-microtubule network. For these reasons, enucleated cells - here referred as cytoplasts - offered an interesting possibility to untangle the geometrical and molecular cues that specifically control centrosome position (Karsenti et al., 1984). Plating them on adhesive micropatterns revealed that centrosome self-positions at the geometrical center of the cytoplasts suggesting that its off-centering in cells is due to microtubule interaction with the nucleus (Dupin et al., 2009; Graham et al., 2018). However, the centrosome often detaches from the nucleus when moving to the cell periphery during the migration of neuroblasts (Umeshima et al., 2007) or epithelium formation (Strzyz et al., 2015) for example. This might indicate that the centrosome-microtubule network could be empowered of active off-centering properties independently of the nucleus, although this has not yet been demonstrated.

Here, we show that actin contractile network plays an important role in the confinement of the microtubule networks while the positioning of the centrosome at the center of this actin-based boundary is achieved by dynein-based forces on microtubules. 


\section{Results}

\section{Centrosome is off-centered in cells plated on anisotropic adhesion pattern}

The centrosome of mouse embryonic fibroblasts (MEF) was located at the geometrical center of cells plated on relatively isotropic adhesive patterns such as equilateral triangles (Figure 1A), as previously described (Hale et al., 2011). However, it appeared shifted from the geometrical center when cells were plated on isosceles triangle, despite the fact that in these conditions cells were not migrating nor forming contact with any adjacent cells (Figure 1B). With that micropattern, the actin-network architecture was polarized, and not just a homothetic transformation of the cell contour. The width of the network was greater along the triangle's base and the arrangement of actin bundles differed between the larger vertices and the smaller apex (Figure 1B). This suggested that the centrosome positioning away from the geometric center could be due to the asymmetry in the actin network. To further investigate this possibility, actin-network asymmetry was re-inforced. Previous work has shown that the distribution of the cell's adhesions to a substrate can direct the architecture of the actin network (Chen et al., 2019; Mandal et al., 2014; Théry et al., 2006b), and this can be achieved by plating a cell on a micropattern such that the cell adopts a convex edge and a concave edge. In this situation, the actin network tends to flow from sites of cell-substrate adhesions at the convex edge, towards sites between cell-substrate adhesion at the concave edge where stress fibers tend to form. Thus, cells were plated on L-shaped or C-shaped micropatterns to impose asymmetric actin network architecture. With both micropatterns, the actin network displayed a marked asymmetry and the centrosome was significantly shifted from the geometric center, in the direction of the actin-network retrograde flow towards the edge harboring contractile stress fibers (Figure 1C, D). Similar shift was observed in human retinal pigment epithelial (RPE1) cells and mouse muscle myoblast (C2C12) (Figure S1). This supported the idea that centrosome positioning is affected by the pattern of cell adhesions and the architecture of the actin network.

However, the nucleus position was also shifted from the cell geometrical center when cells were plated on anisotropic micropatterns (Figure 1E). So it was unclear whether the centrosome was off-centered because it was attached to an off-centered nucleus or whether the microtubule-centrosome network was not self-centering with respect to cell shape in those conditions.

\section{Centrosome is off-centered in cytoplasts plated on anisotropic adhesion pattern}

Cell enucleation allows the study of centrosome-microtubule network interaction with cell peripheral boundary without the bias of the interaction with the nucleus. Cytoplasts, i.e. enucleated cells, were produced by centrifugation of attached cells on ECM-coated plastic slides (Piel et al., 2000). They were then detached and plated on large $2000 \mu \mathrm{m} 2$ disc-shaped micropatterns, in order to maximize their spreading and the available space for centrosome positioning in $2 \mathrm{D}$. However, we found that the major network of vimentin intermediate 
filament in fibroblasts and RPE1 cells forms a dense network around the nucleus (Patteson et al., 2019), which can resist enucleation, maintain its perinuclear architecture and affect microtubule network organization (Figure S2B). To avoid any geometrical bias due to intermediate filaments we further worked with vimentin-KO MEF (Patteson et al., 2019). In these cells, centrosomes were found to precisely position at the cell geometrical center of isotropical shapes: $84 \%$ were found in a $5 \mu \mathrm{m}$ wide region at the center of the disc (Figure $2 \mathrm{~A}$ ) or of equilateral triangles (Figure 2B). On discs, centrosomes displayed similar centering efficiency in cells and cytoplast, with or without the vimentin network (Figure S2C).

In order to investigate centrosome positioning in anisotropic conditions, we plated cytoplasts expressing EGFP-Centrin1 on a variety of triangular geometries. We first chose triangles of similar area but different height to bases ratio: equilateral, short isosceles (ratio 7/4) and isosceles (ratio 9/2) (Figure 2B, C and Figure S3). Thousands of different geometrical centers have been described in triangles (Kimberling). We measured centrosome position relative to some centers that are interesting because their definition presumes simple relationship with the sides or the vertexes of the triangle. Those are the circumcenter (equidistant to the triangle vertexes), the incenter (equidistant to the triangle sides), the geometrical center (which reflects the entire area of the triangle) and the orthocenter -which minimizes the sum of its distances to the triangle vertexes and to those of its pedal triangle. The distances between these centers increase with the height to base ratio of the triangle (Figure S3A), reflecting the variations of the contributions of their definition parameters (distance to vertex, distance to sides, distance to the middle of sides). To evaluate centrosome positioning, a triangular contour was fitted to the edges of the plated cytoplast (Figure S3B) to evaluate the position of all centers of the triangles (Figure S3C). With a greater height-to-base ratio, the centrosome was more distant from all pre-defined centers except the geometric center, or center of mass, to which it remained in close proximity (Figure S3D,E). This was in agreement with the accepted understanding of centrosome positioning, in that the entire area of the cytoplast, ie the entire cell mass, was implicated in its positioning, rather than the cell periphery alone (Kimura and Kimura, 2011). The robustness of the prediction that the centrosome is positioned at the geometric center was confirmed in cytoplasts plated on more exotic geometries (Figure S4).

\section{The centrosome sits at the center of the actin network}

Interestingly, the centrosome and the geometric center were found to be separated by a small distance in the cytoplasts plated on short isosceles triangles (Figure 2C), as it was observed in nucleated cells (Figure 1). To further explore the conditions leading to centrosome off-centering cytoplasts were plated on L-shaped or C-shaped micropatterns shapes to impose asymmetric actin network architecture. With both micropatterns, the actin network was asymmetric and, as in the case of nucleated cells, the centrosome was significantly shifted from the geometric center, away from actin transverse arcs and towards peripheral stress fibers (Figure 2D). A similar shift was observed in cytoplast obtained from C2C12 (Figure S5). This supported the idea that centrosome positioning is affected by the 
pattern of cell adhesions and the architecture of the actin network, independently of the position of the nucleus.

Interestingly, we noted that a central zone in the cell was devoid of actin bundles, an observation that has been made by others as well (Dong et al., 2019), and we termed this region the Actin Inner Zone (AIZ). We manually detected the contour of this zone and its geometrical center: the Actin Inner Center (AIC) (Figure 3A). Our measurements revealed that the centrosome was closer, or equally distant, to the AIC than to the cell geometrical center in all the conditions we tested (Figure 3B, see Figure S6 and S7 for representative examples of centrosome positioning with respect to the AIZ). Therefore, the AIC appeared to be a better descriptor of centrosome positioning than the cell geometrical center

\section{The centrosome position adapts to changes in the architecture of the actin network}

The association between the architecture of the actin network and centrosome positioning was further examined by plating cytoplasts on various sizes of disks ranging from 500 to $3000 \mu \mathrm{m} 2$. Surprisingly, we found that the extent of the distribution of centrosome positions was independent of the size of the disk (Figure S8A, B). However, the size of the averaged AIZ was also relatively independent of the size of the disk (Figure S8C) in line with the idea that centrosome positioning was sensitive to the AIZ.

To modulate the shape and position of the AIZ, cytoplast were plated on short isosceles triangles as those shapes were shown to shift centrosome position away from the geometric center (Figure 2C), and because cytoplast spreading was more efficient on triangles than on L- or C-shapes. Accurate analysis of centrosome positioning was not practical through chemically inhibiting actin assembly, Arp $2 / 3$ or formin because of the detachment of cytoplasts from adhesive micropatterns in those conditions. However, inhibiting Rho kinase ROCK with Y27632 resulted in a regular, homogeneous and homothetic network of thin and loose actin bundles along all cell edges (Figure 4A). In particular, the width of the network along the short edge of the triangle was lower than in the control condition, and was similar to those along the two longer edges (Figure 4B,C). In cytoplasts treated with the ROCK inhibitor, the center of the AIZ, the AIC, and the centrosome positioned in close proximity to the geometric center, unlike in the control condition, where the AIC and centrosome were positioned further from the short edge of the triangle than the geometric center (Figure 4D and 4E). Noteworthy, in cells displaying a poorly contractile actin network, no transverse arcs and, therefore, no asymmetric AIZ like PtK2 cells, the centrosome was found precisely at the cell geometrical center (Figure 4F), as in MEF cytoplasts treated with ROCK inhibitor (Figure 4E). Altogether these results showed that the actomyosin network acts as a spatial boundary for the microtubule network and thereby affects centrosome position

\section{Microtubules orientation adapts to the architecture of the actin network}

Centrosome positioning is known to depend on the mechanical forces exerted on the microtubule network (Kimura and Kimura, 2011; Wu et al., 2011). We thus investigated 
whether microtubules inside of the AIZ engaged specific and distinct interactions from those on the outside. To quantify how microtubule organization is effected by the AIZ in comparison with the actin-dense region (termed the Actin Peripheral Zone; APZ), we analyzed local variations in microtubule morphologies and orientations in MEF cytoplast plated on disc-shaped micropattern (Figure 5A, 5B, see examples of segmented networks in Figure S9). The orientations were clustered into two categories; radial $\left(<45^{\circ}\right)$ and tangential $\left(>45^{\circ}\right)$, in relation to the angle in which the microtubule at a given point (pixel) crosses a straight line originating from the centrosome. The averaged local orientation was further described by the orientation ratio, which was defined as the ratio of pixels with radial orientations over those with tangential orientations. Based on orientation ratios, microtubules were more radially oriented in the AIZ than in the APZ (Figure 5C). Moreover, by performing a linescan along a cytoplast radius (Figure 5D), we found that the transition between radial to tangential orientations occurred precisely at the transition between the AIZ and the APZ (Figure 5E, see examples of linescans in Figure S10). From these results, we concluded that the architecture of the actin network acts locally on the shape and orientation of microtubules.

\section{Microtubule disassembly perturbs centrosome positioning independently of its impact on actin network contraction.}

Microtubule disassembly is known to impair centrosome positioning (Burakov et al., 2003; Dupin et al., 2009; Hale et al., 2011). Cytoplasts were plated on ice (2 hours) and treated with $10 \mu \mathrm{M}$ nocodazole to induce a complete disassembly of microtubules (Figure 6A, middle). As expected, this treatment induced a dispersion of centrosome positions (Figure 6B, middle). However, and in accordance with previous studies, microtubule depletion also increased cell contractility and induced the formation of large actomyosin bundles (Figure 6B, middle) (Chang et al., 2008; Krendel et al., 2002). The shape of the AIZ was severely deformed and was shifted asymmetrically with respect to the overall cytoplast shape (Figure 6C, middle). The centrosome mis-positioning appeared aligned with the shift of the AIZ (see examples in Figure S11).

The distortion of the AIZ in response microtubule disassembly could also be detected in cytoplasts plated on disks. Centrosome positioning was not completely random throughout the cytoplast, even after 20 hours of nocodazole treatment (Figure S12A, B), but limited to the AIZ (Figure S12C). Therefore, and importantly, both experiments suggested that the wellknown mispositioning of the centrosome in response to microtubule disassembly resulted not only from the absence of microtubules but also from the deformation of actin-based spatial boundaries through increased actin-network contractility.

To counterbalance the increase of contractility associated to microtubule disassembly, high doses of the ROCK inhibitor Y27632 (100 or $200 \mu \mathrm{M})$ were also added to nocodazoletreated cytoplasts (Figure 6A, right). In these conditions, the shape of the AIZ was similar to that in cytoplasts treated with Y27632 alone (at $20 \mu \mathrm{M}$ ); i.e. the AIZ formed a regular, homothetic peripheral band along the cell edges (Figure 6C, right). The lateral shift in the position of the AIZ was less than that in cytoplasts treated with nocodazole alone and the distribution of centrosomes followed the same trend (Figure 6B). Notably, in response to 
either nocodazole alone or nocodazole and Y27632, the centrosome-AIC distances were higher than those in the control cytoplasts (Figure 6D), showing that although centrosome positioning was coordinated with AIZ displacement, centrosomes were dispersed within the AIZ in the absence of microtubules. This suggested that microtubules direct centrosome positioning to the center of the AIZ.

\section{Dyneins position the centrosome at the center of the actin network}

Dyneins have been shown to be involved in centrosome positioning in eggs (Tanimoto et al., 2016), embryos (Nguyen-Ngoc et al., 2007; De Simone et al., 2016), unicellular eukaryotes (Koonce, 1999; Rehberg et al., 2005; Vogel et al., 2009) and mammalian cells (Burakov et al., 2003; Wu et al., 2011). The two dynein inhibitors we tested, ciliobrevin D and dynarrestin, had no clear effect on the dispersion of the Golgi apparatus, which is a classic readout for dynein inactivation. Therefore we chose to inhibit dynein activity by expressing a dominant negative form of the dynactin subunit p150glued (p150-DN) (Wu et al., 2018). For these experiments we worked with WT MEF to detect the GFP signal of p150DN without being perturbed by the centrin1-GFP signal in the vimentin-KO line with used before. In cytoplast of WT MEF plated on isoceles triangle, the centrosome was off-centered relative to cell geometrical center as in vimentin-KO cytoplasts (Figure S13). In dyneininactivated cytoplasts, the centrosomes were more dispersed than in control cytoplasts but still biased towards the apex of the triangle and the position of the AIZ (Figure7A). Similar observations were made upon dynein inactivation in RPE1 cytoplasts (Figure S14). In these conditions, microtubules were curvy all over the cell (Figure 7B) and the networks were highly asymmetric (Figure S14). However, the position of the centrosome remained limited by the actin bundles (Figure 7B) suggesting that dyneins were involved in centrosome positioning with respect to this network.

To further test this hypothesis we used the same experimental strategy as before to anihilate actin network asymmetry by relaxing acto-myosin contractility. In dyneininactivated cytoplasts treated with the ROCK inhibitor Y27632, centrosomes were then dispersed all over the cell and around the geometric center, corresponding to the shifted position of the AIZ (see the positions and angular distributions of centrosome in Figure 7A second and fourth columns). This showed that dyneins directed centrosome positioning to the center of the AIZ rather than to the geometric center of the cell.

To better understand how dyneins could direct centrosome position at the center of the AIZ, we performed a co-labelling of actin filaments, microtubules and p150glued (Figure 7C). p150Glued labeling revealed that dyneins were not evenly distributed throughout the entire cell and were concentrated within the AIZ (Figure 7D). Furthermore, the variations of size and symmetry of the AIZ were correlated to the same changes in the spatial distributions of p150Glued (Figure S15). This suggested that, in addition to the peripheral pushing forces exerted by contractile actin bundles deforming microtubules out of the AIZ (Figure 5), microtubules were put under higher tension in the AIZ by the concentrated distribution of dyneins in this region. 


\section{Conclusion}

\section{Centrosome, nucleus and the cell geometrical center.}

The mechanism which specifically regulates the positioning of the centrosome in mammalian cells has long been confused with the mechanism that regulates the position of the nucleus, and notably the actomyosin network acting on the nucleus (Burakov and Nadezhdina, 2013; Burke and Roux, 2009; Luxton and Gundersen, 2011). The consensus has been that the centrosome positions at the cell's geometric center, either autonomously (Dupin et al., 2009; Graham et al., 2018) or in association with the nucleus (Hale et al., 2011). Here, we established an in vitro cell system to study centrosome positioning in which a number of parameters were controlled. This system included cytoplasts devoid of nuclei and the major intermediate filament, vimentin, and cytoplasts of defined shapes dictated by the micropatterned substrates onto which they were plated. We showed that the centrosome position is defined by the architecture of the actin network. It is located at the center of the actin network; more precisely, it is positioned at the geometric center of an inner space that is devoid of actin bundles. This position can correspond or not to the cell's geometric center, depending on the anisotropy of the actin network, which in turn depends on the geometry of cell's adhesion.

Furthermore, our experiments in nucleated cells showed that centrosome off-centering is also active in cells but that it can be perturbed by the mechanism of nucleus positioning. In poorly contractile cells like PtK2, the centrosome distribution was well clustered around cell geometrical center and the nucleus distribution was spread around it (Figure 4). By contrast, in more contractile cells like fibroblasts, RPE1 cells or C2C12 myoblast, the distribution of centrosomes were more dispersed than the distribution of nuclei (Figure S1), and the distribution of centrosomes were more clustered in cytoplast than in cells (Figure S5); suggesting that the nucleus position is strongly determined by the contraction of the actomyosin network and that the nucleus can displace the centrosome from its position at the center of the actin network. These data showed that both organelles have independant selfcentering properties (Reinsch and Gönczy, 1998; Vallee and Stehman, 2005) and that the level of acto-myosin contraction defines which of the two will dominate the competition.

Microtubule and actin.

Microtubules interact with actin via specific crosslinkers or non-specific steric interactions (Dogterom and Koenderink, 2018). In particular, a dense and growing actin network can apply pushing forces on microtubules (Colin et al., 2018; Gupton et al., 2002). Here, we found that the actomyosin network constitutes the actual spatial boundary to which the microtubules are sensitive. Disrupting or modifying the geometry of the actin network via the pattern of cell adhesions, altered the spatial boundary and changed centrosome position accordingly (Figure 2, 3, 4). Microtubules appeared more bent within the actomyosin network at the cell periphery, and straighter in the central part devoid of actin (Figure 5). These results suggested that centrosome positioning is mainly ensured by a combination of peripheral pushing and central pulling forces along microtubules. 
Dyneins.

Dynein have long been known to apply pulling forces on microtubules and to be thus involved in MTOC positioning. By acting at the periphery or throughout the cytoplasm in round eggs, dyneins are thought to position the centrosome at the egg geometrical center (Kimura and Kimura, 2011). We found here that in adherent mammalian cells, the activity of dynein is not evenly distributed all over cell periphery but rather restricted to specific regions of the cell cortex. Indeed, the linear shape and radial orientation of microtubules and the increased amount of dynein within the central part of the basal cortex devoided of actin bundles suggest that dyneins put microtubules under tension in a subcellular pattern defined by the geometry of the contractile actin network (Figure 4B-E,7C, Figure S10). Consistent with this view, the microtubules are not able to interact with cell periphery in aged cytoplasts but the centrosome remains well positioned, suggesting that the inner part of the cell cortex is sufficient to ensure centrosome position at the cell center (Brodsky et al., 2007). Dynein activity requires dynactin to be coupled to a cargo or any other substrate supporting the force load. In large and round embryos, dyneins have been proposed to act as a coupling device that transmits contractile forces from the actomyosin network onto microtubules (De Simone et al., 2016). Whether this applies to spread cells, and how it sets and organizes tensional forces in these conditions where the microtubule network lies along the actin network remains to be investigated.

Implications for centrosome positioning in differentiated/polarized cells.

The centrosome-positioning forces mediated via the actin network appeared potent and relevant for the cell in vivo because highly asymmetric actin networks, such as those developed on $\mathrm{C}$-shaped micropatterns, brought the centrosome into contact with cell periphery, potentially reflecting the peripheral positioning mechanism in migrating or polarized cells (Bornens, 2018; Tang and Marshall, 2012). Hence, a change in actin contractility and actin-network asymmetry (due to changes in adhesion geometry) could be the initial step affecting the organization of microtubules and the distribution of dyneins, which, by repositioning the centrosome, could further bias internal traffic and reinforce the directional bias of the cell's polarization. 


\section{Acknowledgements}

We thank Robert Goldman for sharing the MEF WT and vimentin-KO cell lines, Hiroki Umeshima for sending us the plasmid p150glued DN, Franck Perez, Sandrine Moutel and the plateform TabIP for sharing their electroporator and antibodies, the cytometry plateforme of the Institute for Research of Saint-Louis Hospital, as well as Tanguy Chocat and Esteban Beuve Helissey for their assistance in performing experiments. This work was supported by the European Research Council (ERC Adv 741773 to L.B., ERC CoG 771599 to M.T.) and a post-doctoral fellowship from La Ligue contre le cancer (FFJL14157 to A.J.J.).

\section{Author contributions}

Conceptualization : M.T., L.B., M.P., M.B.; Funding Acquisition, M.T., L.B., A.J.J.;

Methodology : M.T., L.B., M.P., M.B., A.J.J., A.S.; Investigation : A.J.J., A.S., C.D., G.L., B.V.; Analysis : M.T., L.B., A.J.J., G.L.; Supervision : M.T., L.B. Writing : M.T., A.J.J..

\section{Declaration of interest}

The authors declare no conflict of interest. 


\section{Methods}

\section{Cell Culture}

Cell culture, cell lines, plasmids and transfection and drug treatment.

MEF WT and KO for Vimentin cell lines (received from Robert Goldman), C2C12 and PTK2 cells were cultured in Dulbecco's Modified Eagle Medium (31966, Gibco) supplemented with 10\% FBS (50900, Biowest) and 1\% antibiotic-antimycotic (15240-062, Gibco). RPE1 cells were cultured in Dulbecco's Modified Eagle Medium Nutrient Mixture F-12 (31331-093, Gibco) supplemented with 10\% FBS and 1\% antibiotic-antimycotic. MEF KO Vimentin EGFP-Centrin 1 were made by transient transfection of pEGFP-C1-Centrin1 (kindly provided by James Sillibourne) with lipofectamine LTX (15338100, Invitrogen) in Opti-MEM (11058, Gibco) according to the procedure described by the manufacturer. Selection was performed with G418 at $0.5 \mathrm{mg} / \mathrm{ml}$ and sorted by FACS twice with one month interval. They were posteriorly cultured with $0.2 \mathrm{mg} / \mathrm{ml}$. For p150 inhibition assay on cytoplasts, we used WT MEF instead of vimentin KO MEF to avoid confusion between GFP from centrin1 and from the p150 construct, or RPE1 cells. Centrosome positioning was similar in both cells lines (Supplemental Figure S13). Cells were electroporated with NEPA21 electroporator (from Nepa Gene) with the plasmid expressing GFP-p150-CC1 (214-548 aa of p150Glued) obtained from Mineko Kengaku (Kyoto University) and according to the manufacturer's protocole for MEF cells. Cells were then sorted by FACS $24 \mathrm{~h}$ after electroporation and plated directly on slides for enucleation and enucleated $48 \mathrm{~h}$ after electroporation. Living cells were incubated and imaged at $37^{\circ} \mathrm{C}$ with $5 \% \mathrm{CO} 2$ in a humidified environment.

\section{Enucleation}

Cells were seeded the night before, $12 \mathrm{~h}$ before enucleation on RINZL plastic micro-slides (71890-01, Delta Microscopies) precoated with Collagen I Rat Protein, Tail (A1048301, Gibco) at $12 \mu \mathrm{g} / \mathrm{ml}$ and Fibronectin from bovine plasma (F1141, Sigma) at $1 \mu \mathrm{g} / \mathrm{ml}$ for 1 hour. Cells were seeded to achieve a $90 \%$ confluence by the time of the enucleation. Cells were put on $50 \mathrm{ml}$ tubes resistant to high-speed centrifugation $(339652$, Nunc) in complete medium with Cytochalasin D (C8273, Sigma) at $3 \mu \mathrm{g} / \mathrm{ml}$ for $30 \mathrm{~min}$ at $37^{\circ} \mathrm{C}$, then centrifuged at $15^{\prime} 000$ $\mathrm{g}$ for $1 \mathrm{~h}$ at $37^{\circ} \mathrm{C}$. Cytoplasts were then washed twice with pre-warmed DMEM then let them to rest for $30 \mathrm{~min}$ at $37^{\circ} \mathrm{C}$ before detachment for seeding on micropatterns. An alternative protocole was used for $\mathrm{C} 2 \mathrm{C} 12$ cells. In this case, cells were seeded the night before on RINZL slides pre-coated with Fibronectin (at $10 \mu \mathrm{g} / \mathrm{ml}$ for $40 \mathrm{~min}$ ). On the day of the experiment, cells were incubated with Cytochalasin $\mathrm{D}$ at $2 \mu \mathrm{g} / \mathrm{ml}$ for $45 \mathrm{~min}$ at $37^{\circ} \mathrm{C}$, then centrifuged at $10^{\prime} 000 \mathrm{rpm}$ at $37^{\circ} \mathrm{C}$ using an ultra-centrifuge (Avanti JXN-26, Beckman Coulter) equipped with a swinging rotor (JS-13.1, Beckman Coulter).

\section{Drug treatment}

Microtubules were removed by incubating cells in HBSS (14025092, Gibco) on ice and in a cold room at $4^{\circ} \mathrm{C}$ for 2 hours then warmed up to $37^{\circ} \mathrm{C}$ in complete medium with $10 \mu \mathrm{M}$ Nocodazole (M1404, Sigma) and incubated until fixation. Rock inhibition was achieved with 
Y27632 (Y0503, Sigma) at $20 \mu \mathrm{M}$. Rock inhibition in the absence of microtubules was achieved with cold incubation as described above and warming up with complete medium with $10 \mu \mathrm{M}$ Nocodazole and Y27632 at 100 or $200 \mu \mathrm{M}$ as specified for $2 \mathrm{~h}$ at least.

\section{Fixation and Immunostaining}

Cells plated on coverslips were fixed with Paraformaldehyde (15710, Euromedex), Glutaraldehyde (G5882, Sigma) or a mixture of both depending on the antibodies used. All fixation mixtures were done in Cytoskeleton Buffer supplemented with Sucrose (CBS, see below) with $0.1 \%$ Triton X-100 (T8787, Sigma) with either 3\% Paraformaldehyde, 3\% Paraformaldehyde $+0.025 \%$ Glutaraldehyde or $0.5 \%$ Glutaraldehyde. Fixation mixture was added to the cells for $10 \mathrm{~min}$ at room temperature. Glutaraldehyde related autofluorescence was quenched with a solution of PBS and $1 \mathrm{mg} / \mathrm{ml}$ sodium Borohydride for $10 \mathrm{~min}$ at room temperature. Cells were then re-permeabilised with Triton $0.1 \%$ in PBS for $10 \mathrm{~min}$ at room temperature, then blocked with Bovine Serum Albumin (BSA, A2153, Sigma) at 1.5\% in PBS for 10 min. Antibodies were diluted in PBS containing 1.5\% BSA and both incubation with primary or secondary antibodies was made for 1 hour. Microtubules were stained with MCA77G from Abd serotec, ab18251 from Abcam or A-R-H\#02 from Tab-IP (Curie Institute antibody platform). Centrosome staining was performed with anti-gamma Tubulin (T6557,Sigma), anti-pericentrin (ab4448, Abcam ; 611815, BD Biosciences) or antipolyglutamylated Tubulin (A-R-H\#04, TabIP platform, Institut Curie). Vimentin was stained with $5741 \mathrm{~S}$ from Cell Signaling using methanol fixation for $5 \mathrm{~min}$ at $-20^{\circ} \mathrm{C}$. Dynein was stained with anti-p150glued antibodies (612709, BD Biosciences). A particular protocole was used for Dynein staining with an extra step of pre-permeabilisation using $0.025 \%$ Triton X100 in CBS for 30 seconds followed by fixation in 4\% PFA $+0.05 \%$ Glutaraldehyde $+0.1 \%$ Triton X-100 in CBS for $10 \mathrm{~min}$. Actin filaments were stained with Phalloidin-A555 (A34055, Life Technologies) or Phalloidin-A568 (A12380, Life Technologies) together with secondary antibodies. Staining with DAPI (D9542, Sigma) was performed systematically with secondary antibodies to stain the nucleus or to control proper enucleation. Coverslips were mounted with Mowiol 4-88 (81381, Sigma).

Cytoskeleton Buffer supplemented with Sucrose (CBS): A stock solution containing $10 \mathrm{mM}$ HEPES (H3375, Sigma) at pH 6.1, 138 mM KCl (P3911, Sigma), 3 mM MgCl2 (208337, Sigma) and 2 mM EGTA (E3889, Sigma) was made. Sucrose was added extemporaneously before use at $0.32 \mathrm{M}(10 \%)$.

\section{Micropatterning}

\section{Micropattern prototyping using Primo}

The micropatterning protocol was adapted from (Strale et al., 2015).

Micropattern were obtained by shining a pattern of UV light through the microscope objective on a PEGylated glass coverslip covered with a liquid containing a photo-initiator. To limit the volume of liquid to use for the process (here choose at $30 \mu \mathrm{l}$ ), a custom silicone chambers was assembled using two sandwiched $250 \mu \mathrm{m}$ silicon sheets, cut with a plotter-cutter 
(GRAPHTEC CE-6000-40). For the bottom layer a millimeter pear shape in-between inlet and outlet channels ( 0.5 um wide), and for the top layer, holes upward the end of the inlet and outlet channels. 18x18 SCHOTT NEXTERION Coverslips \#1.5H "High Performance" were used for prototyping steps. Coverslips were handles under a laminar flux hood. To promote attachment of PDMS chambers then attachment of Poly-1-Lysine-Polyethylenglycol/PLLPEG, the surface of the coverslips was oxidized 40 seconds by exposure to air-plasma (PE-30, Plasma Etch) at $30 \mathrm{~W}$, under vacuum and with an air flow rate of $10 \mathrm{cc} /$ minute. Then the silicone chamber was put onto the activated side followed by the introduction of PLL-PEG solution from the inlet channel. The PLL-PEG solution (PLL(20)-g[3.5]-PEG(2), SurfaceSolutionS, Switzerland), at a concentration of PLL-PEG at $0.1 \mathrm{mg} / \mathrm{ml}$ in $10 \mathrm{mM}$ HEPES at pH7.4, was incubated for 30 minutes at room temperature. This step was performed extemporaneously, right before patterning. The coverslip was avoided to dry after this step and during and between all following steps. The coverslip was washed once with of PLPP (4benzoylbenzyl-trimethylammonium chloride, $14.5 \mathrm{mg} \mathrm{ml}-1$ ) then fresh PLPP was added, by removing most of the liquid at the outlet with a highly absorbant precision paper (Kimwipes, Kimtech). Surface patterning was performed right after on an inverted Nikon microscope Ti-E equipped with a CFI Super Plan Fluor 20× ELWD (NA 0.45) objective capable of high UVtransmission, a Perfect Focus System 3, an ORCA-Flash 4.0 LT CMOS camera (Hamamatsu), a motorized stage (Märzhäuser) and the Primo module containing a DMD illumination with a $375 \mathrm{~nm}(4.5 \mathrm{~mW})$ laser (Alvéole Lab). The microscope was controlled with the ImageJ $\mu$ manager software and the Primo module was controlled with the $\mu$ Manager-Leonardo plugin (Alvéole Lab). Micropattern shapes were designed using ImageJ, as an 8bit image with patterns filled in white on a black background. Each field was exposed for 25 seconds at $100 \%$ UV power corresponding to a $900 \mathrm{~mJ} / \mathrm{mm} 2 \mathrm{UV}$ energy dose. The coverslips were washed 3 times with PBS by pipetting 100ul in the inlet while aspirating at the outlet using a high absorbant precision paper to create a flow within the chamber. Coverslips were then incubated with Fibronectin at $20 \mu \mathrm{g} / \mathrm{ml}$ in PBS for 20 min by flowing $100 \mu$ l of the solution, and then washed 3 times with PBS as described above. The silicone chamber assembly can be detached at this step by immersing coverslips in PBS and using tweezers. Patterned sample were kept at $4^{\circ} \mathrm{C}$ in PBS and used the next day. Cells were plated as described bellow for deep UV patterning.

\section{Large scale micropatterning with deep UV and cell seeding}

The micropatterning protocol was adapted from (Azioune et al., 2010).

Polystyrene coating : 20x20 Coverslips (1304369, Schott) were cleaned for 10min in acetone then for $10 \mathrm{~min}$ in isopropanol in a bath sonicator and then dried with compressed-clean air under a laminar flow hood. They were coated with adhesion promoter Ti-Prime (MicroChemicals) using a spin-coater (WS-650m2-23NPPB, Laurell) at $3000 \mathrm{rpm}$ for 30s and baked on top heater for $2 \mathrm{~min}$ at $120^{\circ} \mathrm{C}$. Then a $1 \%$ polystyrene (MW 260,000, 178891000, Acros Organic) solution in toluene (179418, Sigma) was spin-coated on the coverslip at $1000 \mathrm{rpm}$.

Plasma treatment and micropatterning : Polystyrene layer was oxidized by exposure to airplasma as described above to promote the attachment of PLL-PEG to the surface, which was diluted as described above and incubated for $30 \mathrm{~min}$ at room temperature. PLL-PEG was 
removed and coverslips room air dried before putting them in tight contact with a chromed printed photomask (Toppan Photomask). Tight contact was maintained using a vacuum holder. The PLL-PEG layer was burned with deep UV $(\lambda=190 \mathrm{~nm})$ through the non-chromed windows of the photomask, using UVO cleaner (Model No. 342A-220, Jelight), at a distance of $1 \mathrm{~cm}$ from the UV lamp with a power of $6 \mathrm{~mW} / \mathrm{cm} 2$, for $4 \mathrm{~min}$.

Cell seeding: Coverslips were washed once with distilled water then incubated with a solution of 40 $\mu \mathrm{g} / \mathrm{ml}$ Fibronectin (F1141, Sigma) in PBS (14190169, GIBCO) for 30min at room temperature. Coverslips were then washed, in a sterile 6-well dish with one coverslip per well and under the laminar flow hood, 3 times with $3 \mathrm{ml}$ sterile PBS, once with $3 \mathrm{ml}$ DMEM and once with 3ml DMEM-10\%FBS-1\%Antibiotic-Antimycotic (complete medium). Cells/cytoplasts were detached with TrypLE (12605036, Gibco), centrifuged and resuspended in complete medium at 100'000 cells $/ \mathrm{ml}$. Most medium was removed for each well containing a coverslip and $1 \mathrm{ml}$ of cell suspension was added. Cells were left for spreading for 1 hour at $37^{\circ} \mathrm{C}$ before washing-out non-attached cells with pre-warmed complete medium. Cells were incubated for at least one more hour at $37^{\circ} \mathrm{C}$ to promote correct spreading and polarisation, before further treatments.

\section{Imaging}

\section{Microscopy}

Most fixed and fluorescently labelled cells were imaged using an up-right epi-fluorescence microscope (Olympus up-right BX61 equipped with a CoolSnapHQ2 camera) monitored by Metamorph. Samples were scanned for cell selection with dry objectives 10x or 20x using a Metamorph plugin developped by Céline Labouesse and Benoît Vianay. Cells were chosen so that they were well spread on sharp patterns and that they do not had a nucleus in the case of conditions with cytoplasts. Cells were imaged with a 100x NA 1.4 oil objective, with $0.5 \mu \mathrm{m}$ spacement between $\mathrm{z}$ planes in a range of $15 \mu \mathrm{m}$. When patterned cells did not fit in one camera field, overlapping images were taken for further stitching. An inverted spinning disk microscope (Nikon Ti2 equipped with a Retiga R3 camera), monitored by Metamorph was used for Figure 7C and Figure S2B using a 60X objective.

\section{Image analysis}

For patterned cells that could not fit in one camera field, ImageJ macros using Stitching plugin were used. Images were then processed the same than single images.

Centrosome positioning analysis was performed with homemade ImageJ suite of macros. The closest plane to the coverslip (cell bottom) was determined creating a band ROI on the actin image, as an expansion of a rough cell border determined by threshold filtering. This ROI was applied to the microtubule channel where the z-plane with the highest Standard Deviation within the band was chosen as cell bottom. Cell Top was determined using the standard deviation of the whole image. Firsts and lasts superfluous z-planes were that way removed to lighten calculations. 
Threshold filtering in actin cell bottom plane was performed to determine cell edges and centroid was calculated. Similar method was used to determine nucleus edges and centroid in the case of conditions with nucleated cells. Scanning the Prominence parameter of the "Find Maxima" plugin from imageJ was performed to determine the prominence value where the number of found maxima was closest to one. Scanning for maxima within the region around this principal centriole was performed to find eventual extra-centrioles. Cells with more than 4 centrioles were discarded. The centroid of the polygon defined after connecting all centrioles 3 by 3 into triangles and adding all areas was used for the calculation of the distances to the cell, and nucleus if applicable, centroids.

All steps contained a quick-scanning verification and assisted-correction module to make sure the analysis was correct for all cells.

\section{Centrosome positioning compared triangle characteristic centres}

In the case of triangle-patterned cytoplasts, the contour defined previously was smoothened by converting curve into a spline defined by a discrete number of close points. The curve defined by the distance of each point from the spline to the previously calculated centroid was smoothened by quadratic regression until the curve presents only 3 maxima, corresponding to the 3 triangle vertexes. The indexes of these three points were used to find the 3 corresponding points the contour-spline. The coordinates of theses 3 points were used to fit the contour of the cell to a triangle. Geometrical calculations were performed to determine the coordinates of 4 characteristic centres of that triangle (centroid, incentre, circumcenter and orthocentre). Distances from the centrosome to these centres were calculated.

\section{Actin inner zone (AIZ) and actin inner centre (AIC)}

Actin inner zone was determined manually on projected and denoised (rolling ball filtering) actin images. The coordinates centroid of the zone was determined and the distance to cell and centrosome centroids was calculated.

\section{Dot plots and plots of AIZs}

An angle correction was determined for all cells in a semi-automatic way. The coordinates of all centres were redressed according to the correction angle and relative coordinates to cell or actin centroid were calculated and plotted. Similar procedure was performed for the regions defining AIZs. Either all contours of AIZs were drawn, or one black 8-bit image was created for each cell and the AIZs was drawn and filled in white. A sum of all the images was made and a Royal LUT was applied.

\section{Microtubule orientations}

Microtubule stacks were skeletonized using a homemade Java plugin. A sum projection was made before a homemade orientation filter was applied to determine the angle made by each pixel of the skeletonized microtubule network. The calculation of a relative angle to the centrosome was performed. This angle corresponds to the angle made locally by a portion of microtubule around a given pixel and the radius defined by the line passing by both the studied pixel and the centrosome. The distribution of relative angle value as a function of the distance to the centrosome was determined and plotted with $\mathrm{R}$. This distribution was also 
performed this time limiting the considered values to a band as shown in the figures or to a given zone like the AIZ.

\section{Statistical Analysis}

Mann-Whitney non-parametric test was used to compare samples using GraphPad Prism software (Version 6.0). Bars in all figures correspond to the median except on Figure 3D. Error bars on Figure 3D correspond to standard error mean (SEM). 


\section{References}

Azioune, A., Carpi, N., Tseng, Q., Théry, M., and Piel, M. (2010). Protein micropatterns: A direct printing protocol using deep UVs. Methods Cell Biol. 97, 133-146.

Biedzinski, S., Agsu, G., Vianay, B., Delord, M., Blanchoin, L., Larghero, J., Faivre, L., Théry, M., and Brunet, S. (2020). Microtubules control nuclear shape and gene expression during early stages of hematopoietic differentiation. EMBO J. 1-15.

Bornens, M. (1977). Is the centriole bound to the nuclear membrane? Nature 270, 80-82.

Bornens, M. (2018). Cell polarity: having and making sense of direction-on the evolutionary significance of the primary cilium/centrosome organ in Metazoa. Open Biol. 8, 180052.

Brito, D.A., Strauss, J., Magidson, V., Tikhonenko, I., Khodjakov, A., and Koonce, M.P. (2005). Pushing forces drive the comet-like motility of microtubule arrays in Dictyostelium. Mol. Biol. Cell 16, 3334-3340.

Brodsky, I.B., Burakov, A. V, and Nadezhdina, E.S. (2007). Microtubules 'Interaction with Cell Cortex is Required for Their Radial Organization, but Not for Centrosome Positioning. Cell Motil. Cytoskeleton.

Burakov, A. V, and Nadezhdina, E.S. (2013). Association of nucleus and centrosome: magnet or velcro? Cell Biol. Int. 37, 95-104.

Burakov, A. V, and Nadezhdina, E.S. (2020). Centering and Shifting of Centrosomes in Cells. Cells 9, 1351.

Burakov, A. V, Nadezhdina, E.S., Slepchenko, B., and Rodionov, V.I. (2003). Centrosome positioning in interphase cells. J. Cell Biol. 162, 963-969.

Burke, B., and Roux, K.J. (2009). Nuclei Take a Position: Managing Nuclear Location. Dev. Cell 17, 587-597.

Burute, M., Prioux, M., Blin, G., Truchet, S., Letort, G., Tseng, Q., Bessy, T., Lowell, S., Young, J., Filhol, O., et al. (2017). Polarity Reversal by Centrosome Repositioning Primes Cell Scattering during Epithelial-to-Mesenchymal Transition. Dev. Cell 40.

Chang, Y., Nalbant, P., Chang, Z., and Bokoch, G.M. (2008). GEF-H1 Couples Nocodazoleinduced Microtubule Disassembly to Cell Contractility via RhoA. Mol. Biol. Cell 19, 2147-2153.

Chen, T., Callan-Jones, A., Fedorov, E., Ravasio, A., Brugués, A., Ong, H.T., Toyama, Y., Low, B.C., Trepat, X., Shemesh, T., et al. (2019). Large-scale curvature sensing by directional actin flow drives cellular migration mode switching. Nat. Phys. 15, 393402.

Chevrier, V. (2002). The Rho-associated protein kinase p160ROCK is required for centrosome positioning. J. Cell Biol. 157, 807-817.

Colin, A., Singaravelu, P., Théry, M., Blanchoin, L., and Gueroui, Z. (2018). Actin-Network Architecture Regulates Microtubule Dynamics. Curr. Biol. 0, 1-10.

Dogterom, M., and Koenderink, G.H. (2018). Actin-microtubule crosstalk in cell biology. Nat. Rev. Mol. Cell Biol.

Dong, L., Gong, J., Wang, Y., He, J., You, D., Zhou, Y., Li, Q., Liu, Y., Cheng, K., Qian, J., et al. (2019). Chiral geometry regulates stem cell fate and activity. Biomaterials 222.

Dupin, I., and Etienne-Manneville, S. (2011). Nuclear positioning: mechanisms and functions. Int. J. Biochem. Cell Biol. 43, 1698-1707.

Dupin, I., Camand, E., and Etienne-Manneville, S. (2009). Classical cadherins control nucleus and centrosome position and cell polarity. J. Cell Biol. 185, 779-786.

Garzon-Coral, C., Fantana, H.A., and Howard, J. (2016). A force-generating machinery maintains the spindle at the cell center during mitosis. Science (80-. ). 352, 11241127. 
Gomes, E.R., Jani, S., and Gundersen, G.G. (2005). Nuclear movement regulated by Cdc42, MRCK, myosin, and actin flow establishes MTOC polarization in migrating cells. Cell 121, 451-463.

Graham, D.M., Andersen, T., Sharek, L., Uzer, G., Rothenberg, K., Hoffman, B.D., Rubin, J., Balland, M., Bear, J.E., and Burridge, K. (2018). Enucleated cells reveal differential roles of the nucleus in cell migration, polarity, and mechanotransduction. J. Cell Biol. 217, 895-914.

Gupton, S.L., Salmon, W.C., and Waterman-Storer, C.M. (2002). Converging populations of f-actin promote breakage of associated microtubules to spatially regulate microtubule turnover in migrating cells. Curr. Biol. 12, 1891-1899.

Hale, C.M., Chen, W.-C., Khatau, S.B., Daniels, B.R., Lee, J.S.H., and Wirtz, D. (2011). SMRT analysis of MTOC and nuclear positioning reveals the role of EB1 and LIC1 in single-cell polarization. J. Cell Sci. 124, 4267-4285.

Karsenti, E., Kobayashi, S., Mitchison, T., and Kirschner, M. (1984). Role of the centrosome in organizing the interphase microtubule array: Properties of cytoplasts containing or lacking centrosomes. J. Cell Biol. 98, 1763-1776.

Kimberling, C. Encyclopedia of Triangle Centers.

Kimura, K., and Kimura, A. (2011). A novel mechanism of microtubule length-dependent force to pull centrosomes toward the cell center. Bioarchitecture 1, 74-79.

Koonce, M.P. (1999). Dynein motor regulation stabilizes interphase microtubule arrays and determines centrosome position. EMBO J. 18, 6786-6792.

Krendel, M., Zenke, F.T., and Bokoch, G.M. (2002). Nucleotide exchange factor GEF-H1 mediates cross-talk between microtubules and the actin cytoskeleton. Nat. Cell Biol. 4, 294-301.

Laan, L., Pavin, N., Husson, J., Romet-Lemonne, G., van Duijn, M., López, M.P., Vale, R.D., Jülicher, F., Reck-Peterson, S.L., and Dogterom, M. (2012). Cortical Dynein Controls Microtubule Dynamics to Generate Pulling Forces that Position Microtubule Asters. Cell 148, 502-514.

Letort, G., Nedelec, F., Blanchoin, L., and Théry, M. (2016). Centrosome centering and decentering by microtubule network rearrangement. Mol. Biol. Cell 27, 2833-2843.

Luxton, G.W.G., and Gundersen, G.G. (2011). Orientation and function of the nuclearcentrosomal axis during cell migration. Curr. Opin. Cell Biol. 23, 579-588.

Malone, C.J., Misner, L., Le Bot, N., Tsai, M.C., Campbell, J.M., Ahringer, J., and White, J.G. (2003). The C. elegans Hook Protein, ZYG-12, Mediates the Essential Attachment between the Centrosome and Nucleus. Cell 115, 825-836.

Mandal, K., Wang, I., Vitiello, E., Orellana, L.A.C., and Balland, M. (2014). Cell dipole behaviour revealed by ECM sub-cellular geometry. Nat. Commun. 5, 5749.

Nguyen-Ngoc, T., Afshar, K., and Gönczy, P. (2007). Coupling of cortical dynein and G alpha proteins mediates spindle positioning in Caenorhabditis elegans. Nat. Cell Biol. 9, 1294-1302.

Patteson, A.E., Vahabikashi, A., Pogoda, K., Adam, S.A., Mandal, K., Kittisopikul, M., Sivagurunathan, S., Goldman, A., Goldman, R.D., and Janmey, P.A. (2019). Vimentin protects cells against nuclear rupture and DNA damage during migration. J. Cell Biol. 218, 4079-4092.

Pavin, N., Laan, L., Ma, R., Dogterom, M., and Jülicher, F. (2012). Positioning of microtubule organizing centers by cortical pushing and pulling forces. New J. Phys. 14.

Piel, M., Meyer, P., Khodjakov, A., Rieder, C.L., and Bornens, M. (2000). The Respective Contributions of the Mother and Daughter Centrioles to Centrosome Activity and Behavior in Vertebrate Cells 7. Cell 149, 317-329. 
Pinot, M., Chesnel, F., Kubiak, J.Z., Arnal, I., Nedelec, F., and Gueroui, Z. (2009). Effects of confinement on the self-organization of microtubules and motors. Curr. Biol. 19, 954-960.

Pitaval, A., Senger, F., Letort, G., Gidrol, X., Guyon, L., Sillibourne, J., and Théry, M. (2017). Microtubule stabilization drives 3D centrosome migration to initiate primary ciliogenesis. J. Cell Biol. 216.

Pouthas, F., Girard, P., Lecaudey, V., Ly, T.B.N., Gilmour, D., Boulin, C., Pepperkok, R., and Reynaud, E.G. (2008). In migrating cells, the Golgi complex and the position of the centrosome depend on geometrical constraints of the substratum. J. Cell Sci. 121, 2406-2414.

Rehberg, M., Kleylein-Sohn, J., Faix, J., Ho, T.-H., Schulz, I., and Gräf, R. (2005). Dictyostelium LIS1 Is a Centrosomal Protein Required for Microtubule/Cell Cortex Interactions, Nucleus/Centrosome Linkage, and Actin Dynamics. Mol. Biol. Cell 16, 2759-2771.

Reinsch, S., and Gönczy, P. (1998). Mechanisms of nuclear positioning. J. Cell Sci. 2295, 2283-2295.

Rodriguez-Fraticelli, A.E., Auzan, M., Alonso, M. a, Bornens, M., and Martin-Belmonte, F. (2012). Cell confinement controls centrosome positioning and lumen initiation during epithelial morphogenesis. J. Cell Biol. 198, 1011-1023.

Salpingidou, G., Smertenko, A., Hausmanowa-Petrucewicz, I., Hussey, P.J., and Hutchison, C.J. (2007). A novel role for the nuclear membrane protein emerin in association of the centrosome to the outer nuclear membrane. J. Cell Biol. 178, 897-904.

Sanchez, A.D., and Feldman, J.L. (2016). Microtubule-organizing centers: from the centrosome to non-centrosomal sites. Curr. Opin. Cell Biol. 1-9.

Shao, W., Yang, J., He, M., Yu, X., Lee, C.H., and Yang, Z. (2020). Centrosome anchoring regulates progenitor properties and cortical formation. Nature.

De Simone, A., Nedelec, F., and Gönczy, P. (2016). Dynein Transmits Polarized Actomyosin Cortical Flows to Promote Centrosome Separation. Cell Rep. 14, 2250-2262.

Starr, D. a, and Fridolfsson, H.N. (2010). Interactions between nuclei and the cytoskeleton are mediated by SUN-KASH nuclear-envelope bridges. Annu. Rev. Cell Dev. Biol. 26, 421-444.

Stinchcombe, J.C., and Griffiths, G.M. (2014). Communication, the centrosome and the immunological synapse. Philos. Trans. R. Soc. Lond. B. Biol. Sci. 369, $20130463-$.

Strale, P.O., Azioune, A., Bugnicourt, G., Lecomte, Y., Chahid, M., and Studer, V. (2015). Multiprotein Printing by Light-Induced Molecular Adsorption. Adv. Mater. 20242029.

Strzyz, P.J., Lee, H.O., Sidhaye, J., Weber, I.P., Leung, L.C., and Norden, C. (2015). Interkinetic Nuclear Migration Is Centrosome Independent and Ensures Apical Cell Division to Maintain Tissue Integrity. Dev. Cell 32, 203-219.

Tang, N., and Marshall, W.F. (2012). Centrosome positioning in vertebrate development. J. Cell Sci. 125, 4951-4961.

Tanimoto, H., Kimura, A., and Minc, N. (2016). Shape-motion relationships of centering microtubule asters. J. Cell Biol. 212, 777-787.

Théry, M., Racine, V., Piel, M., Pépin, A., Dimitrov, A., Chen, Y., Sibarita, J.-B., and Bornens, M. (2006a). Anisotropy of cell adhesive microenvironment governs cell internal organization and orientation of polarity. Proc. Natl. Acad. Sci. U. S. A. 103, 19771-19776.

Théry, M., Pépin, A., Dressaire, E., Chen, Y., and Bornens, M. (2006b). Cell distribution of stress fibres in response to the geometry of the adhesive environment. Cell Motil. Cytoskeleton 63. 
Umeshima, H., Hirano, T., and Kengaku, M. (2007). Microtubule-based nuclear movement occurs independently of centrosome positioning. October 104.

Vallee, R.B., and Stehman, S. a (2005). How dynein helps the cell find its center: a servomechanical model. Trends Cell Biol. 15, 288-294.

Vogel, S.K., Pavin, N., Maghelli, N., Jülicher, F., and Tolić-Nørrelykke, I.M. (2009). Selforganization of dynein motors generates meiotic nuclear oscillations. PLoS Biol. 7, e1000087.

Wu, J., Misra, G., Russell, R.J., Ladd, A.J.C., Lele, T.P., and Dickinson, R.B. (2011). Effects of dynein on microtubule mechanics and centrosome positioning. Mol. Biol. Cell 22, 4834-4841.

Wu, Y.K., Umeshima, H., Kurisu, J., and Kengaku, M. (2018). Nesprins and opposing microtubule motors generate a point force that drives directional nuclear motion in migrating neurons. Dev. 145, 1-10.

Zhang, J., and Wang, Y. (2017). Centrosome defines the rear of cells during mesenchymal migration. Mol. Biol. Cell mbc.E17-06-0366.

Zhang, X., Lei, K., Yuan, X., Wu, X., Zhuang, Y., Xu, T., Xu, R., and Han, M. (2009). SUN1/2 and Syne/Nesprin-1/2 Complexes Connect Centrosome to the Nucleus during Neurogenesis and Neuronal Migration in Mice. Neuron 64, 173-187.

Zhu, J., Burakov, A., Rodionov, V.I., and Mogilner, A. (2010). Finding the Cell Center by a Balance of Dynein and Myosin Pulling and Microtubule Pushing: A Computational Study. Mol. Biol. Cell 21, 4418-4427. 


\section{Figures}

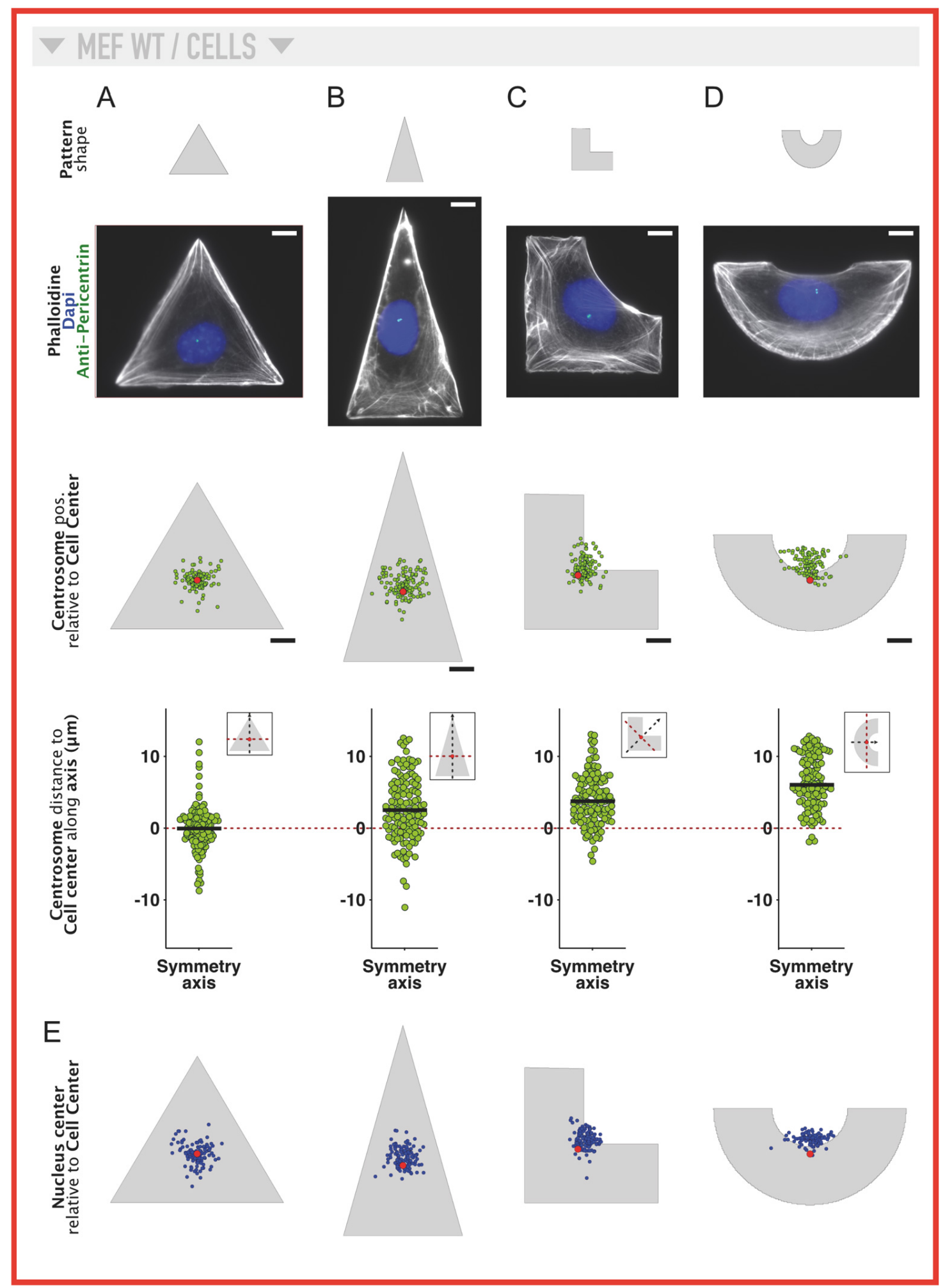

Figure 1. Centrosome is shifted from the geometrical center in cells plated on anisotropic adhesion micropatterns. 
Figure 1. Centrosome is shifted from the geometrical center in cells plated on anisotropic adhesion micropatterns.

A-D MEF WT cells were seeded on $2000 \mu \mathrm{m} 2$ equilateral triangles (A), isosceles "short" triangles (B), L-shapes (C) or C-Shapes (D). Cells were stained for actin using PhalloidineA555 and with anti-Pericentrin to label the centrosome. Graphs show centrosome distribution relative to cell centroid. Plots correspond to the centrosome distance to cell centroid along the axis indicated by dashed black arrows. Dashed red line is perpendicular to black axis and passes by cell centroid. E- Nucleus centroid distributions relative to cell centroid, as indicated. Scale bar : $10 \mu \mathrm{m}$. 


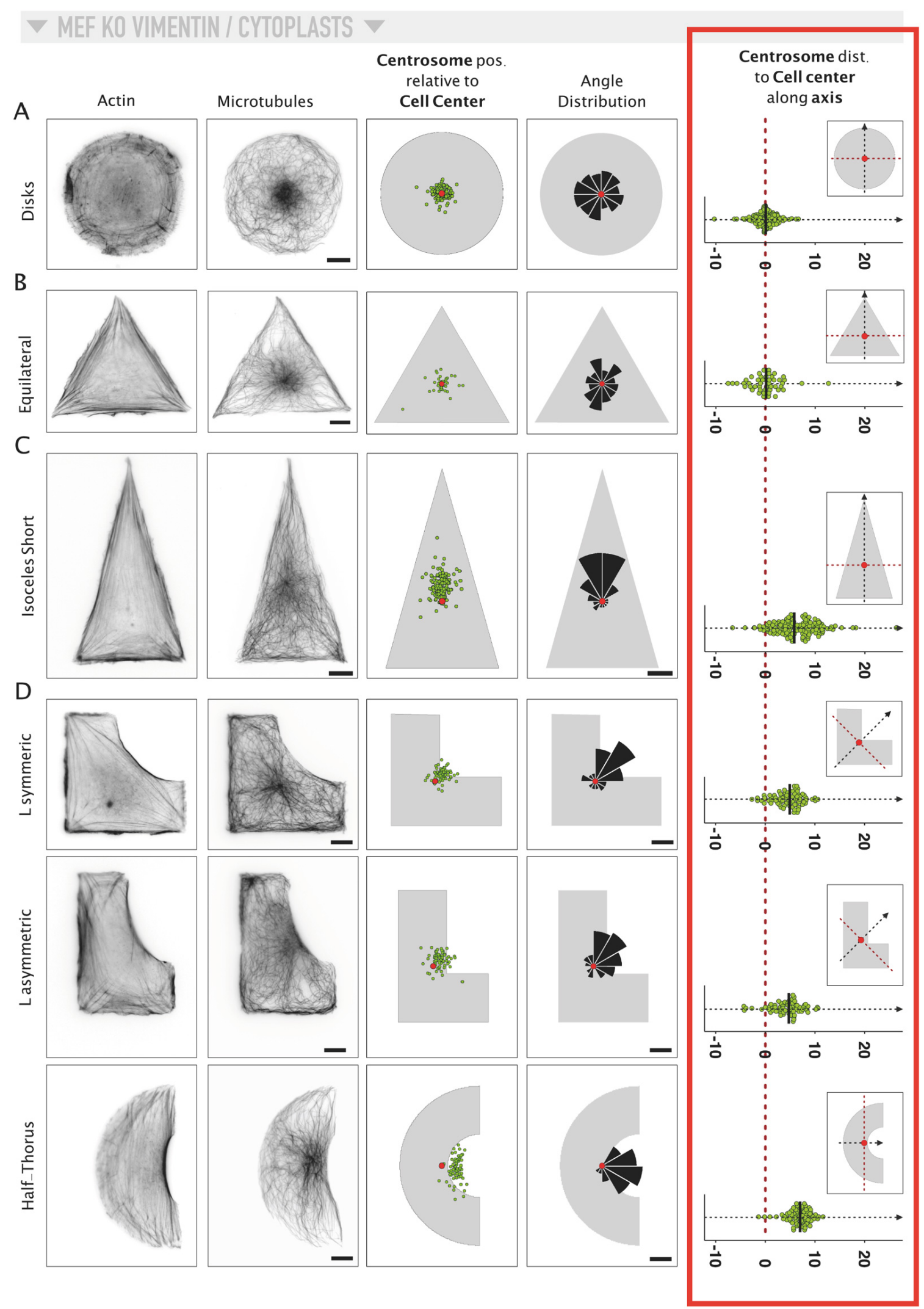

Figure 2. Centrosome positioning at and away from the cell geometrical center in enucleated cells. 
Figure 2. Centrosome positioning at and away from the cell geometrical center in enucleated cells.

A-D Cytoplasts from MEF KO Vimentin cells expressing EGFP-Centrin1 were plated on micropatterns of different shapes with a constant area of $2000 \mu \mathrm{m} 2$ : discs (A), equilateral triangle (B), isosceles triangle 7/4 ratio (C), and anisotropic micropatterns (D), symmetric L, asymmetric $\mathrm{L}$ and $\mathrm{C}$-shapes. For each shape an example of actin and microtubule networks is presented. The plots represent, from left to right, the distribution relative to the cell centroid of centrosome position, the angle distribution and centrosome distance along the axis indicated by dashed-black arrows. Dashed red line is perpendicular to black axis and passes by cell centroid. Scale bars: $10 \mu \mathrm{m}$. 
A

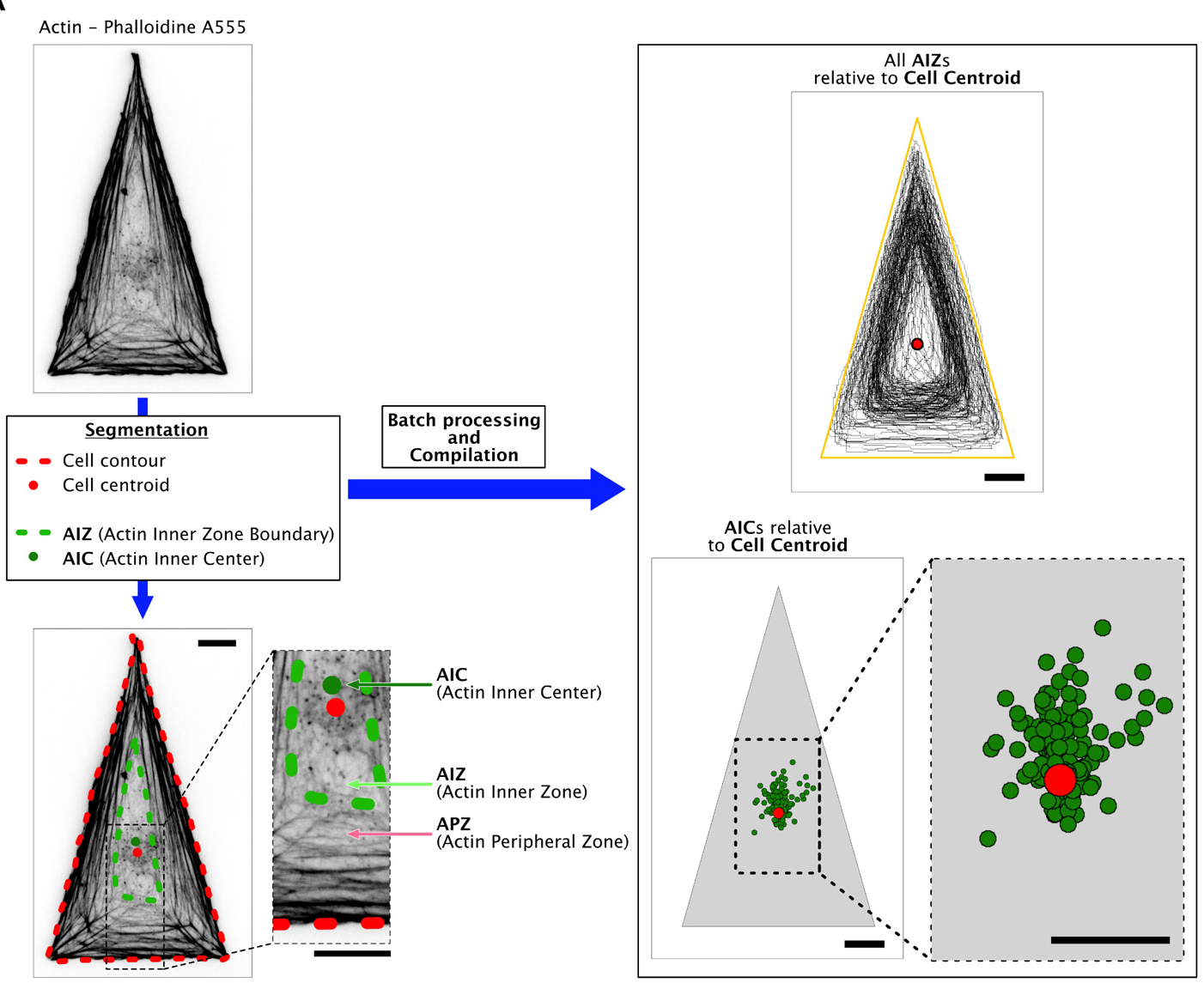

B
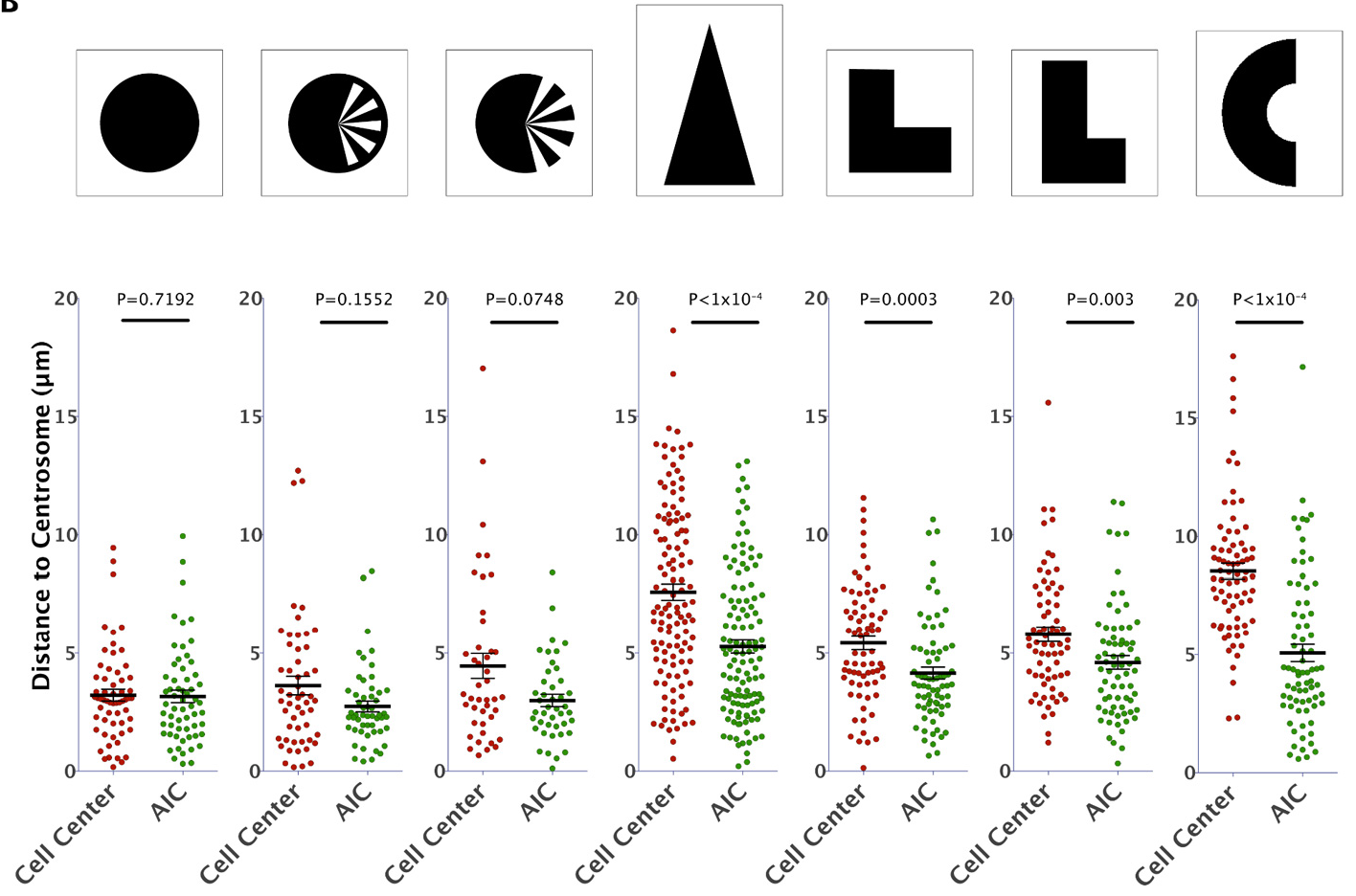

Figure 3. Centrosome positioning close to the actin inner center (AIC). 
Figure 3. Centrosome positioning close to the actin inner center (AIC).

A. Mouse embryonic fibroblast (MEF) vimentin-knockout (KO) cytoplasts expressing EGFPCentrin 1 were plated on short isosceles triangles, fixed and stained with phalloidin-A555. The scheme shows the analysis performed to study the AIZ and AIC. The red dot represents the geometric centers of the cytoplasts and the green dots the AICs.

B. For a variety of shapes, the distance of the centrosome to the cytoplast's geometric center was compared with the distance of the centrosome to the AIC. Scale bars: $10 \mu \mathrm{m}$. 
V MEF KO VIMENTIN / CYTOPLASTS $\nabla$
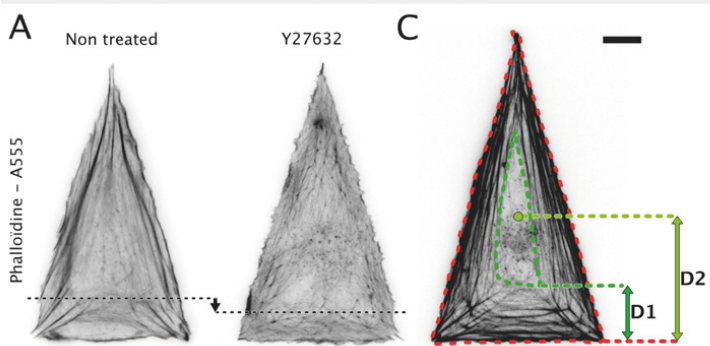

D

Non treated $\quad$ Y27632
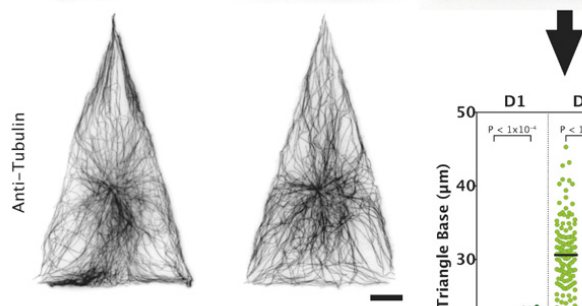

$\downarrow$
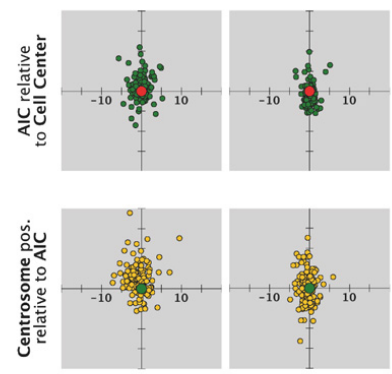

B

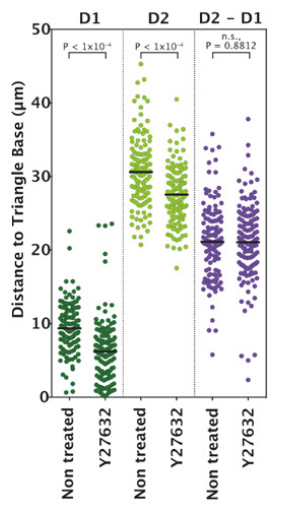

$E$
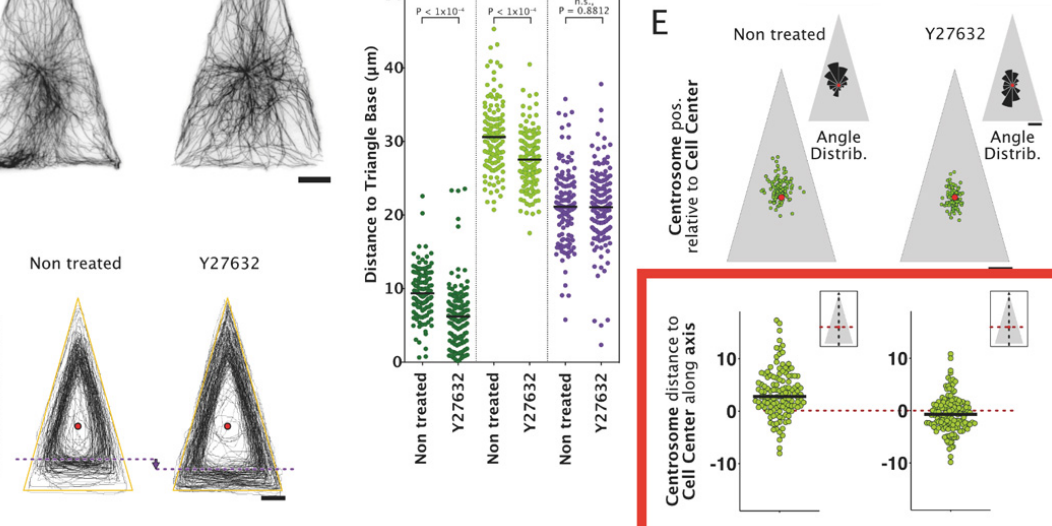

$\mathrm{F}$
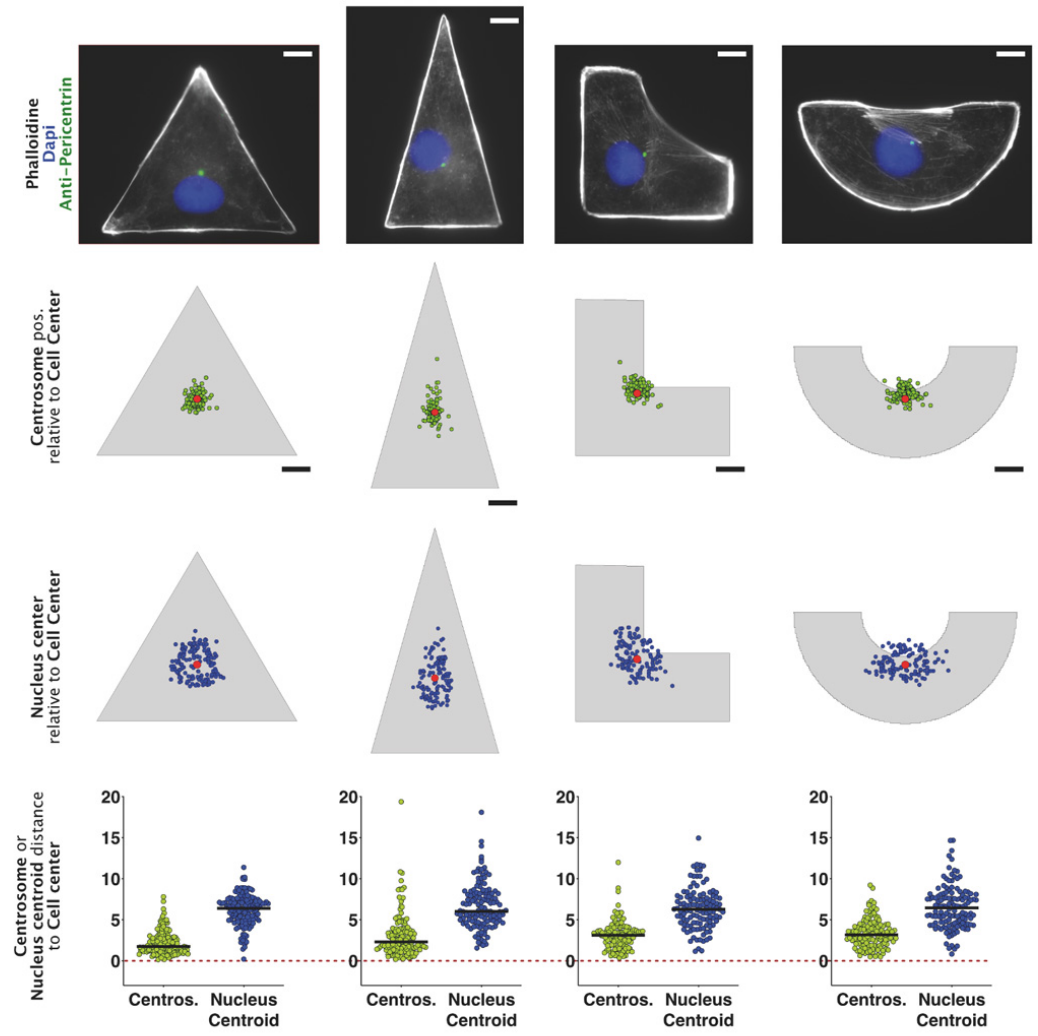

Figure 4. Role of contractility in the architecture of the AIZ and centrosome positioning. 
Figure 4. Role of contractility in the architecture of the AIZ and centrosome positioning.

Cytoplasts plated on $2000 \mu \mathrm{m} 2$ Isosceles "Short" triangles were plated for 2 hours then treated for 2 hours with Y27632 at $20 \mu \mathrm{M}$. Analysis was performed as described before.

A. Microtubule and actin staining as in Figure 1. One representative example is given for each condition.

B. Plots of all the contours of AIZs relative to the cell center.

C. The distance from the centrosome to the triangle basis and the distance from the lowest point of the AIZ to the triangle basis (upper panel) were calculated and plotted (lower panel) as well as the difference between these two distances.

D. Plots of all AICs relative to the cell centroid and of centrosomes relative to the AIC.

E. Plots of all centrosomes relative to the cell center and the respective angle distribution of the population, and its distance to cell centroid along indicated axis.

F. PTK2 cells were platted on isotropic and anisotropic shapes and for each of them one example is given. The plots show centrosome and nucleus centroid distributions relative to cell centroid, and their distance to cell centroid along the axis indicated by dashed-black arrows.

The red dots represents cell geometrical center, the dark-green dots represents the AIC, the light-green and yellow dots represent the centrosome. Blue dots represent the nucleus centroid. Dashed red line represents cell centroid position at the graph origin. Scale bars: $10 \mu \mathrm{m}$. 


\section{MEF KO VIMENTIN / CYTOPLASTS V}

A

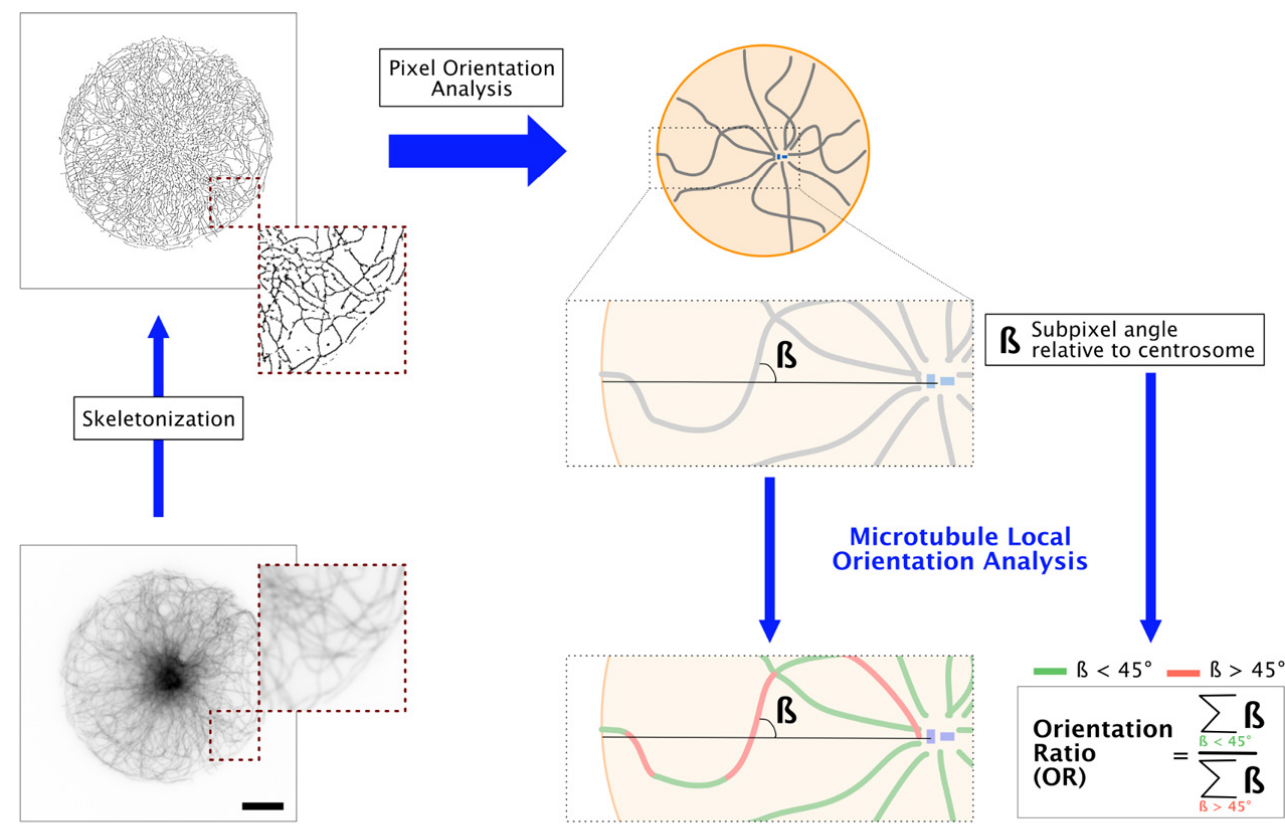

B
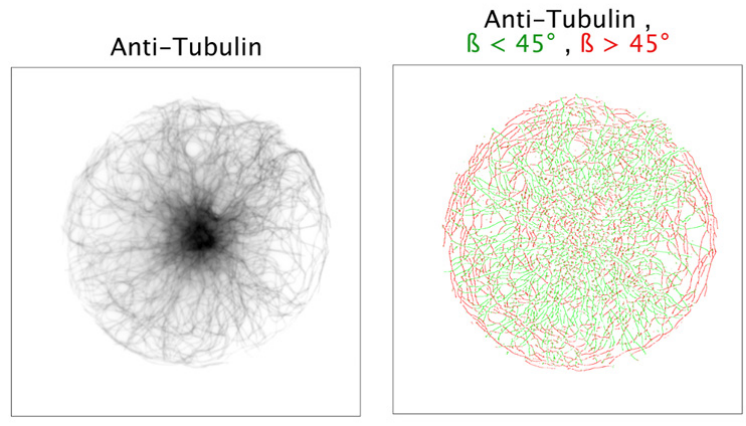

Anti-Tubulin, $ß>45^{\circ}$

Anti-Tubulin, $B<45^{\circ}$
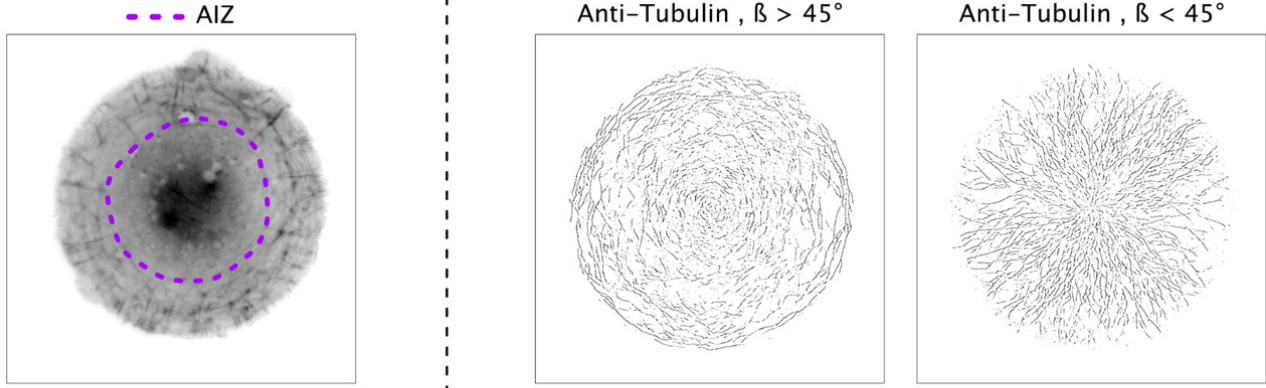

C

D

E
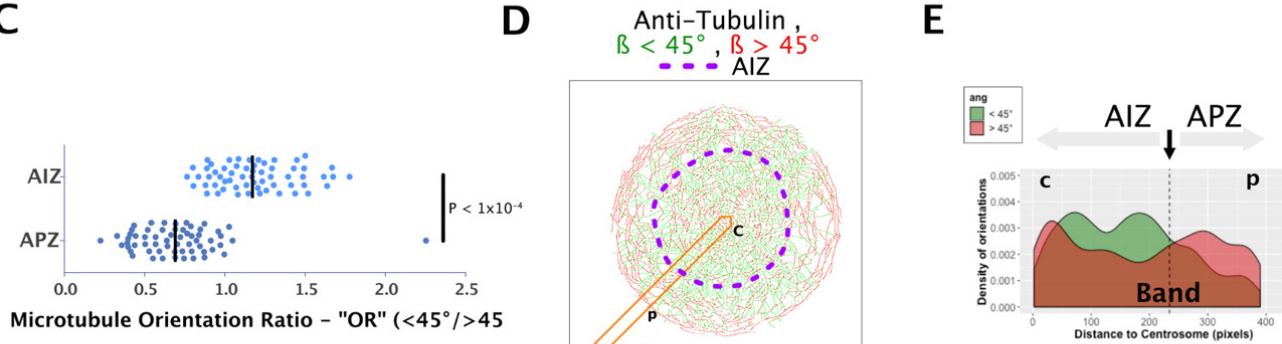

Figure 5. Analysis of microtubule orientation in and outside the AIZ. 
Figure 5. Analysis of microtubule orientation in and outside the AIZ.

Cytoplasts were plated on $2000 \mu \mathrm{m} 2$ disks and stained for Actin and Microtubules like in Figure 1.

A. Overview of the microtubule network analysis. The Orientation Ratio or "OR" is the ratio of the number of non-null pixels with an orientation $<45^{\circ} \mathrm{C}$ and the number of non-null pixels with an orientation $>45^{\circ} \mathrm{C}$.

B. Example of analyzed cytoplast

C. Overview of the microtubule-AIZ cross-analysis: microtubule network analysis was performed along a radial band and in the AIZ and in the APZ.

D. Results of cross-analysis plotting the "OR". The microtubules within AIZ are preferentially oriented radially and the ones outside the AIZ (in the APZ) are preferentially oriented tangentially.

E. The independently determined AIZ boundary matches the boundary of two zones defined by a clear majority of radial or tangential microtubules respectively 
A
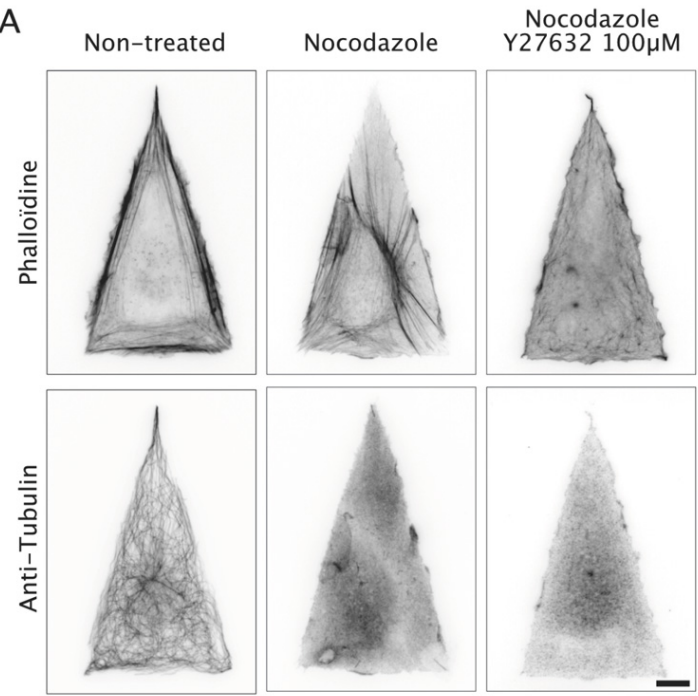

B
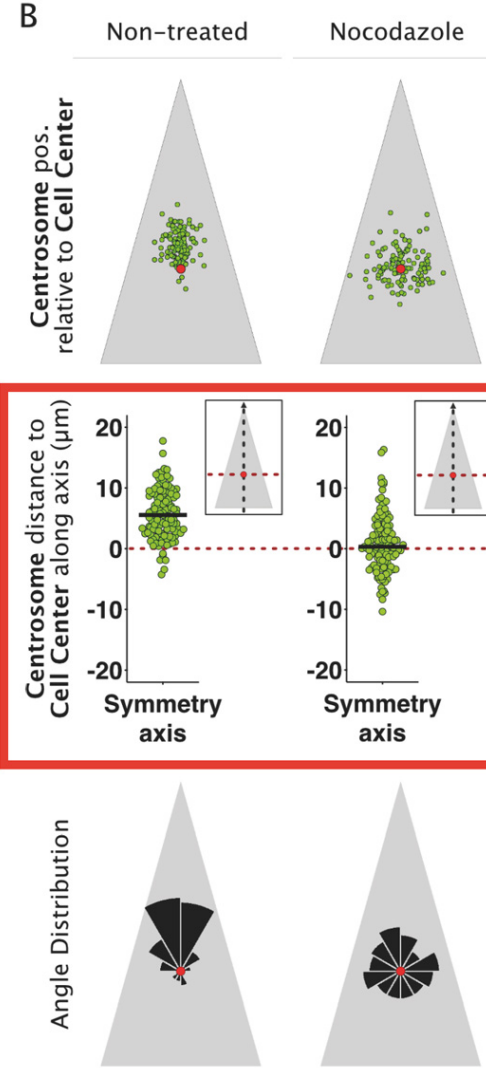

.

嗮,

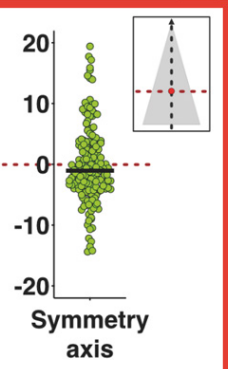

鲜
C

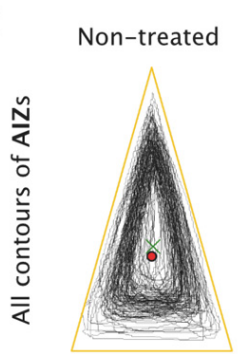

Nocodazole

Nocodazole
Y27632 100u
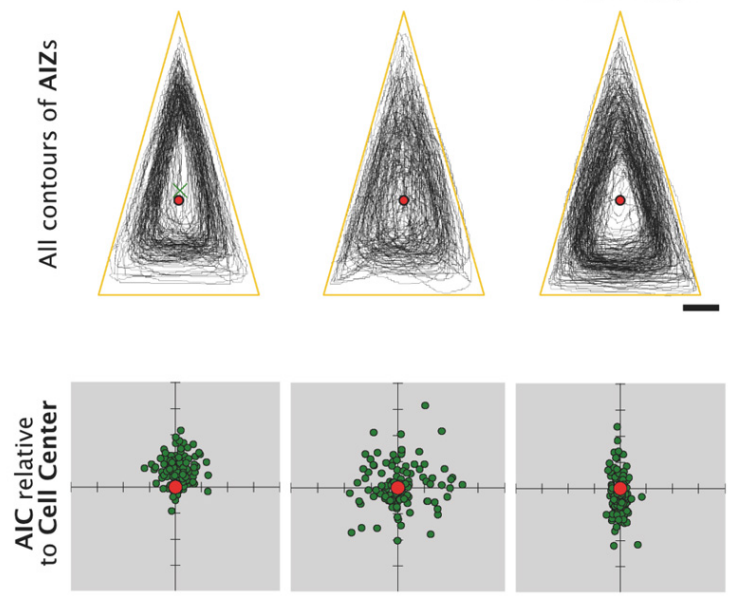

$\mathrm{D}$

Non-treated

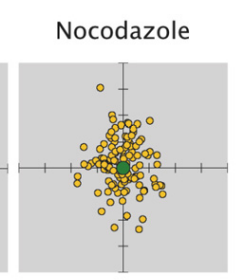

Nocodazole
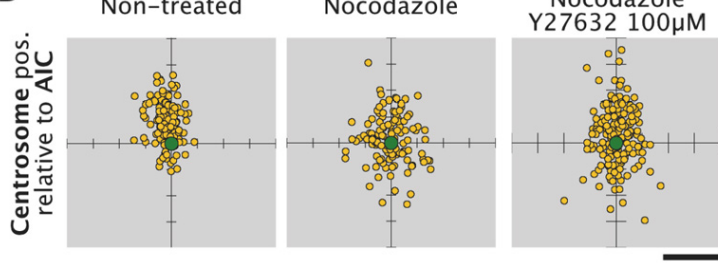

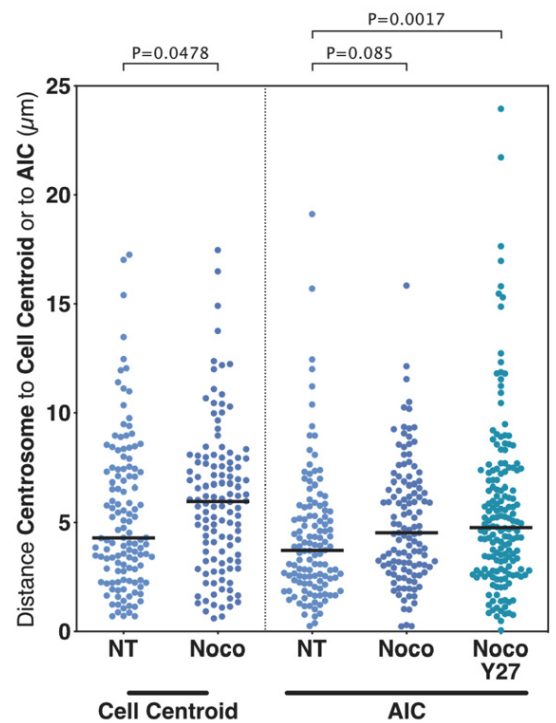

Figure 6. Effect of microtubule disassembly on AIZ and centrosome positioning. 
Figure 6. Effect of microtubule disassembly on AIZ and centrosome positioning.

Cytoplasts plated on $2000 \mu \mathrm{m} 2$ Isosceles "Short" triangles were treated with Ice-Nocodazole $10 \mu \mathrm{M}$ or Ice-Nocodazole $10 \mu \mathrm{M} / \mathrm{Y} 27632100 \mu \mathrm{M}$ in order to uncouple the effect of contractility and the one of microtubules depletion.

A. Representative images of cytoplasts from both conditions. In the absence of microtubules, actin contractility is highly increased. In cells depleted for microtubules and treated with Y27632 at $20 \mu \mathrm{M}$, contractility was still abnormally high (not shown). A higher concentration of Y27632 $(100-200 \mu \mathrm{M})$ was then used. As observed in the example cell, this concentration was sufficient to inhibit contractility in the absence of microtubules.

B. Plots of all centrosomes relative to the cell center, distance to cell centroid along indicated axis and the angle distribution of the population.

C. Plots of all the contours of AICs relative to the cell centroid and of centrosomes relative to the AIC.

D. Plots of all AICs relative to the cell centroid and of centrosomes relative to the AIC.

E. Plot of the distance between the centrosome and the cell centroid or the AIC for the indicated conditions.

Red dots represents cell geometrical center and dark-green dots represent the AIC. The lightgreen and yellow dots represent the centrosome. Scale Bars: $10 \mu \mathrm{m}$. 
$\checkmark$ MEF WT / CYTOPLASTS $\nabla$

A
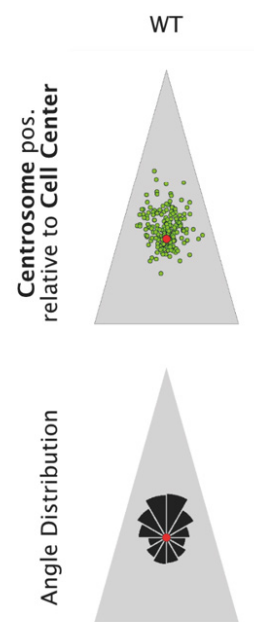
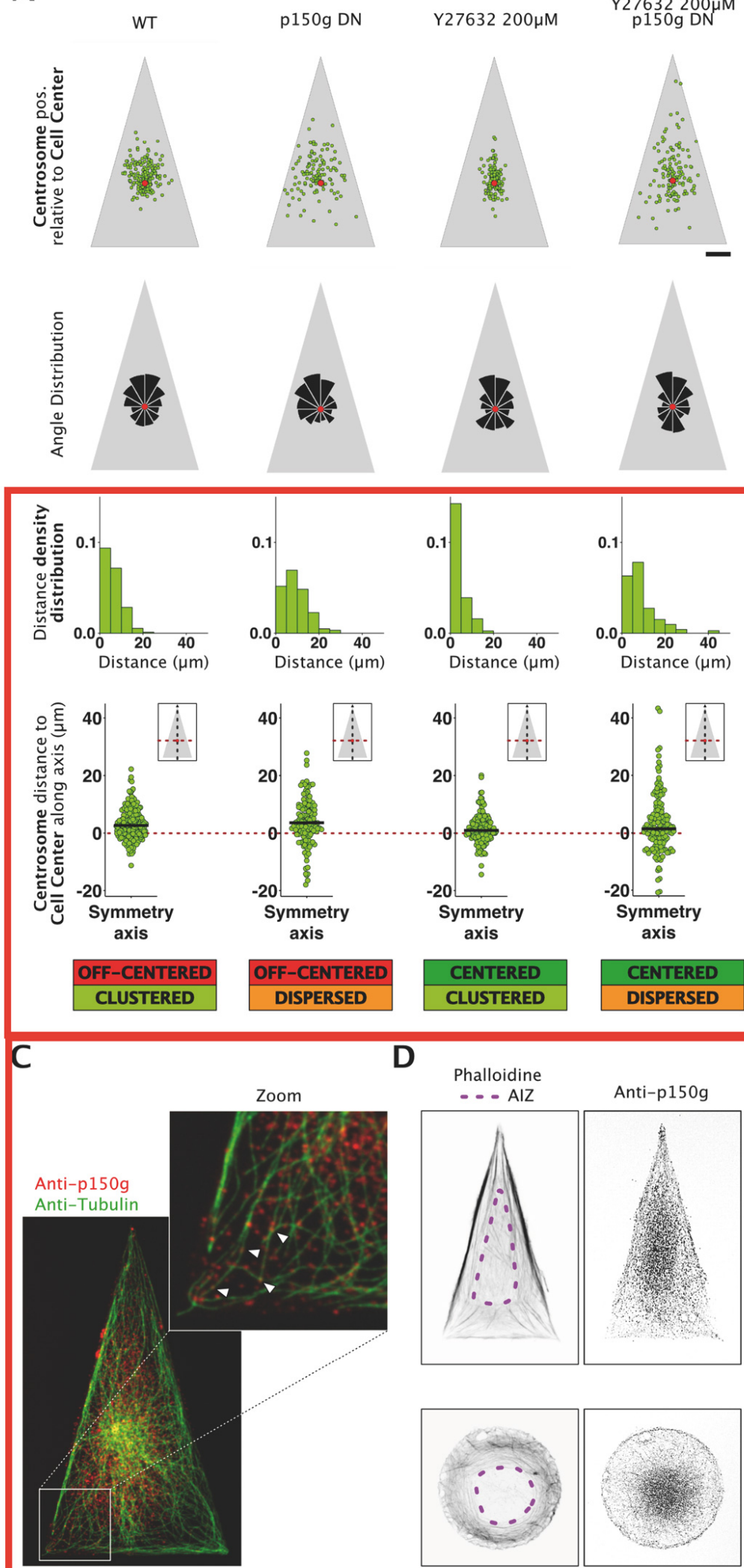

B

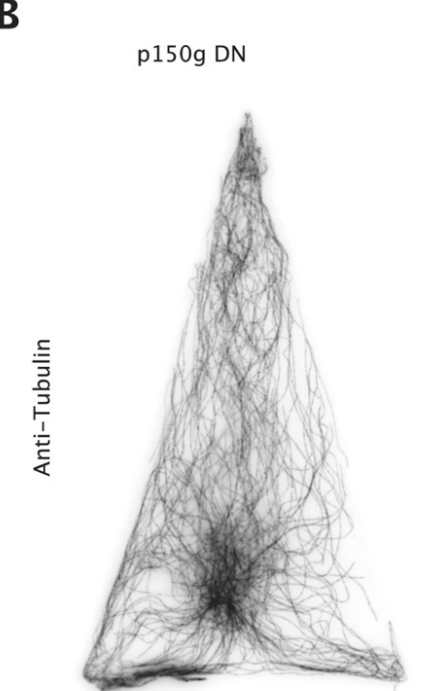

*Gamma $=2$
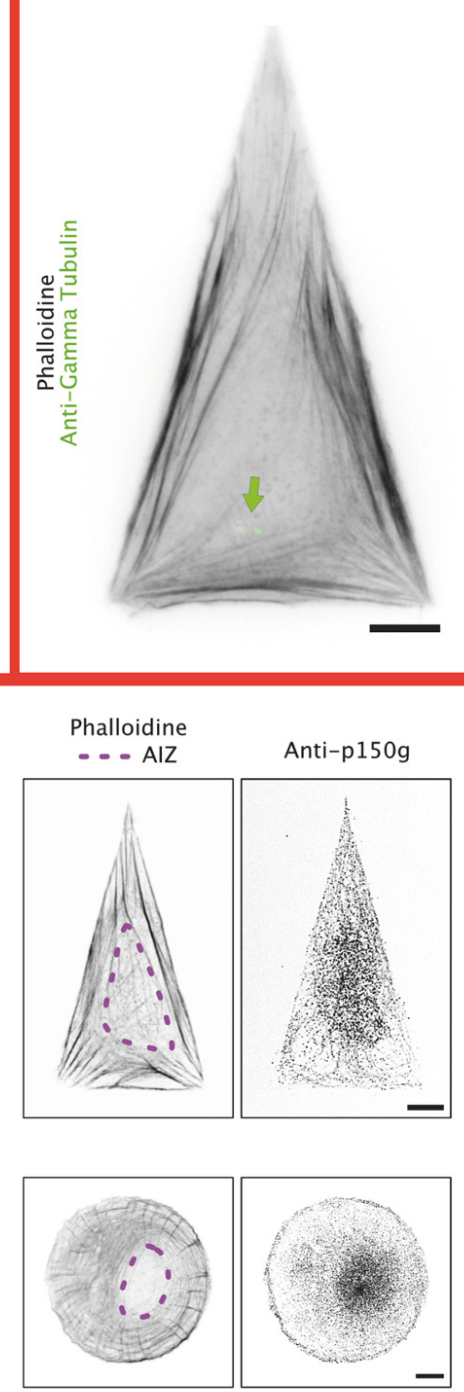

Figure 7. Role of dyneins in centrosome positioning in the AIZ 


\section{Figure 7. Role of dyneins in centrosome positioning in the AIZ}

Cytoplasts of cells electroporated with p150-DN 48 hours before enucleation and positive cells were sorted by flow cytometry $24 \mathrm{~h}$ before enucleation. Cytoplasts were plated on $2000 \mu \mathrm{m} 2$ Isosceles "Short" for 2 hours and treated when indicated with Y27632 at $200 \mu \mathrm{M}$ for 2 extra hours.

A. First line shows the plots of all centrosomes relative to the cell center. Second line the respective angle distribution. Third line shows the density distribution of the centrosome-cell centroid distance and fourth line shows the centrosome-cell centroid distance along the axis indicated by dashed-black arrows. Red dashed line represent the cell centroid position at the origin of the graph.

B. Example of centrosome off-centering in p150g-DN transfected cell.

Red dots represents cell geometrical center and light-green dots represent the centrosome. Green arrow points at the centrosome.

C. Co-staining for microtubules and dyneins. Arrow heads point at dynein dots co-localizing with microtubules.

D. Examples of cytoplasts stained for actin and for dynein using anti-p150glued antibodies. The images were processed with an unsharp mask for better visualization. The AIZ was determined and represented by purple dashed lines. The maximum projection of the dynein images shows a higher overall amount of dynein inside the AIZ. Scale bars: $10 \mu \mathrm{m}$. 


\section{SUPPLEMENTARY FIGURES}

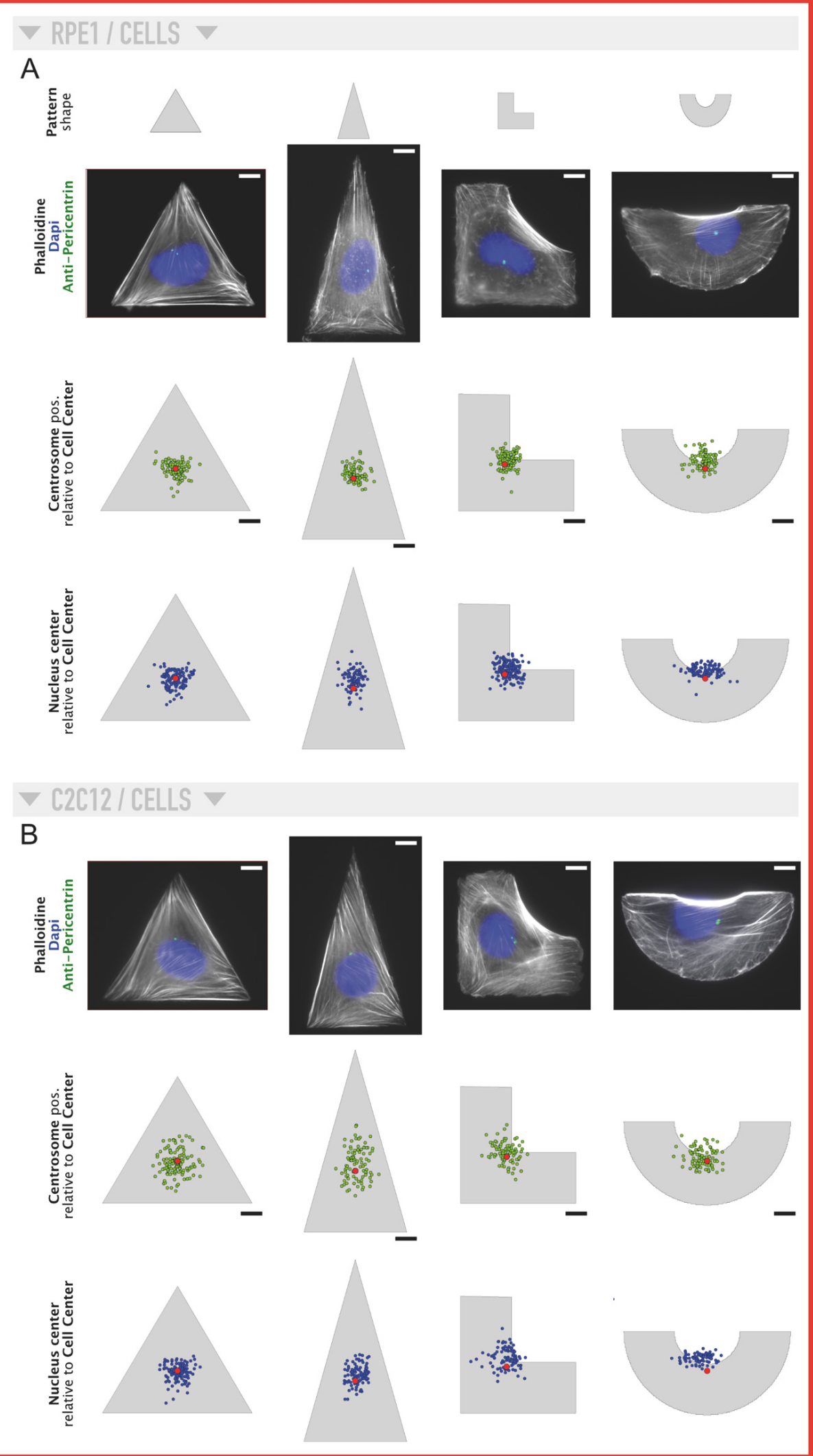

Figure S1. Centrosome is centered in cells plated on isotropic shapes, off-centered in anisotropic shapes. 
A-RPE1 and B-C2C12 cells were seeded on $2000 \mu \mathrm{m} 2$ equilateral triangles, isosceles "short" triangles, L-shapes or U-Shapes. Cells were stained for actin using Phalloidine-A555 and with anti-Pericentrin to label the centrosome. The centrosome position distribution is shifted from the cell centroid in anistropic shapes but centered in isotropic shapes (equilateral triangles). Plots show centrosome and nucleus centroid distributions relative to cell centroid, as indicated. Scale bars: $10 \mu \mathrm{m}$. 
A

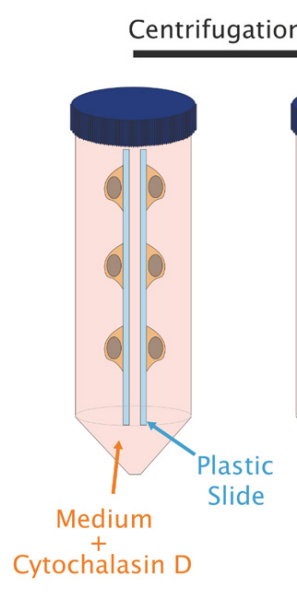

C

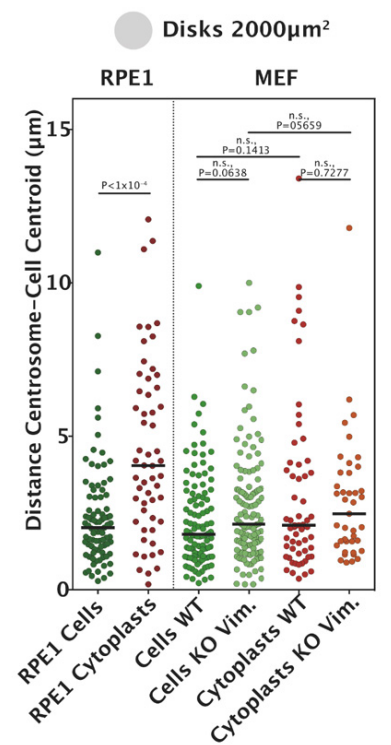

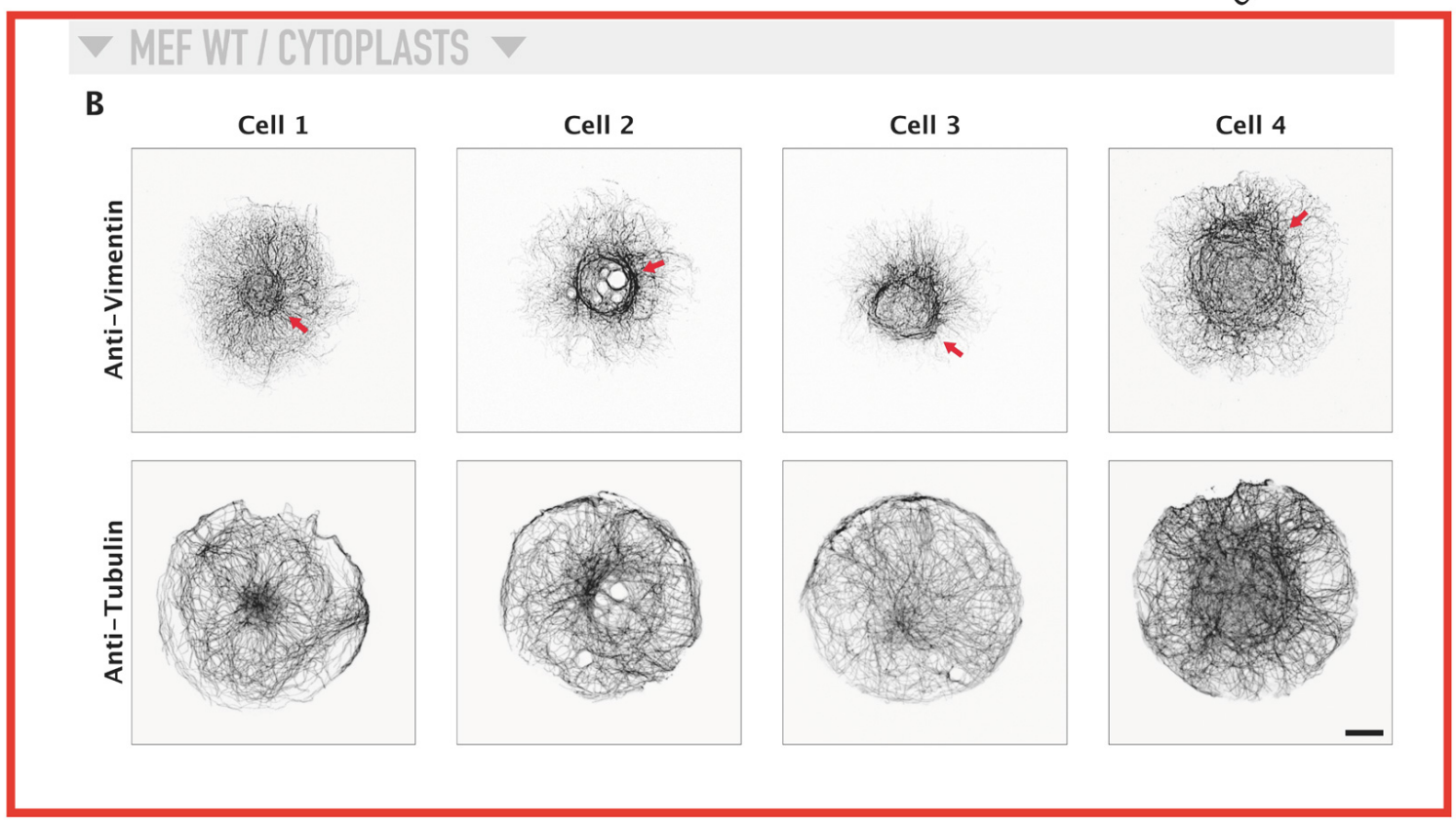

Figure S2. Vimentin filaments bias microtubule organisation in cytoplasts.

A. Cytoplasts protocole overview.

B. Cytoplasts made with MEF WT cells plated on disks (area: $1200 \mu \mathrm{m} 2$, radius: $11.0 \mu \mathrm{m}$ ) and stained with anti-tubulin and anti-vimentin antibodies. An unsharp mask filter was applied to both channels and an enhance contrast was applied to the vimentin images before projection for better visualisation. Cells present Vimentin cage-like structures displacing microtubules, which surround the Vimentin, pointed by red arrows. These cages most likely explain differences in centrosome position distribution between cells and cytoplasts revealed in C. C. Quantification of centrosome position for three cell lines plated in $2000 \mu \mathrm{m} 2$ disks (Radius, $14.2 \mu \mathrm{m}$ ): RPE1, MEF WT or MEF KO for Vimentin. RPE1 cells (which contain high levels of Vimentin) and cytoplasts present very different distribution of centrosome positioning. On the other hand, MEF WT and KO for Vimentin and their respective cytoplasts present very 
similar distribution, also similar to RPE1 cells. The lack of difference between MEF WT and its corresponding cytoplasts may be due to the natural low levels of Vimentin present in MEF WT cells compared to RPE1 cells. Scale bars : $10 \mu \mathrm{m}$. 
A
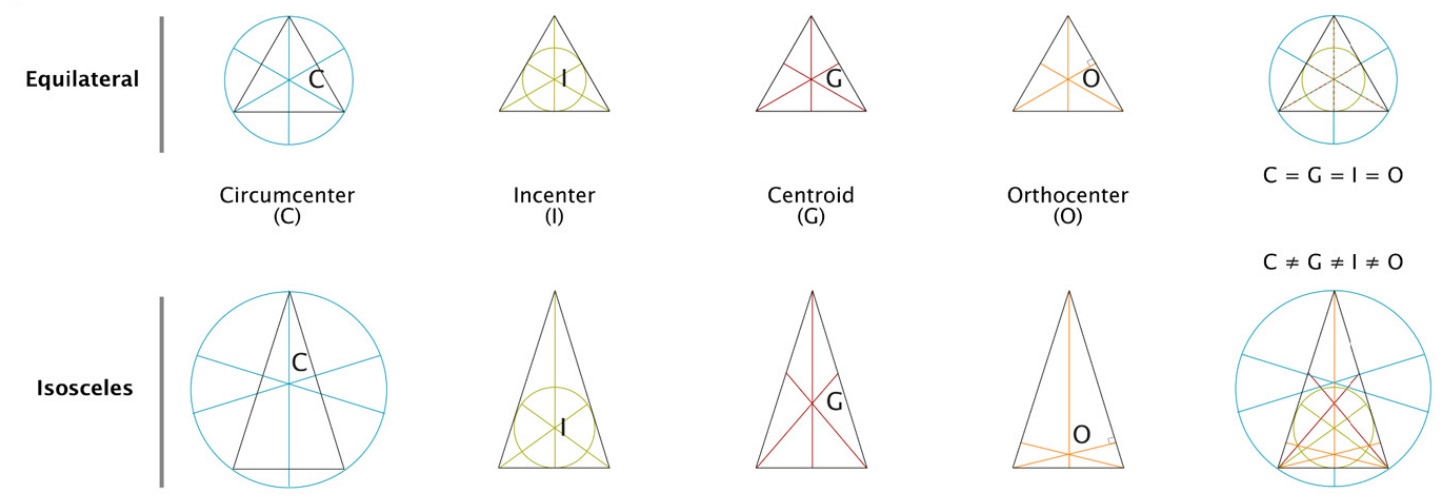

B

Phalloïdine-A555
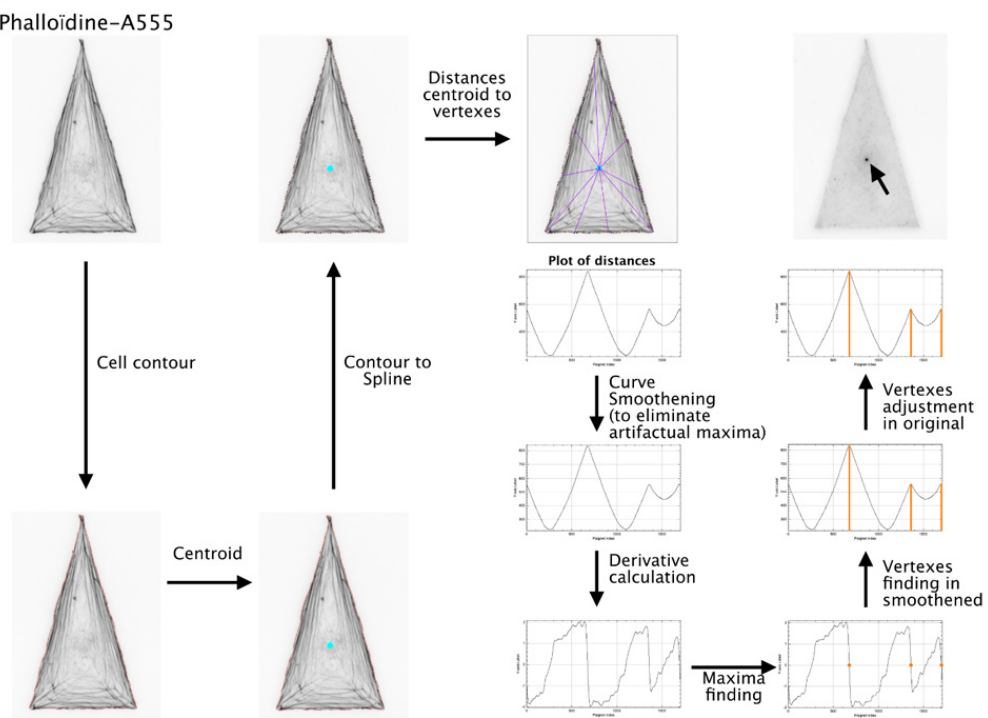

C Triangle analysis and centrosome

D
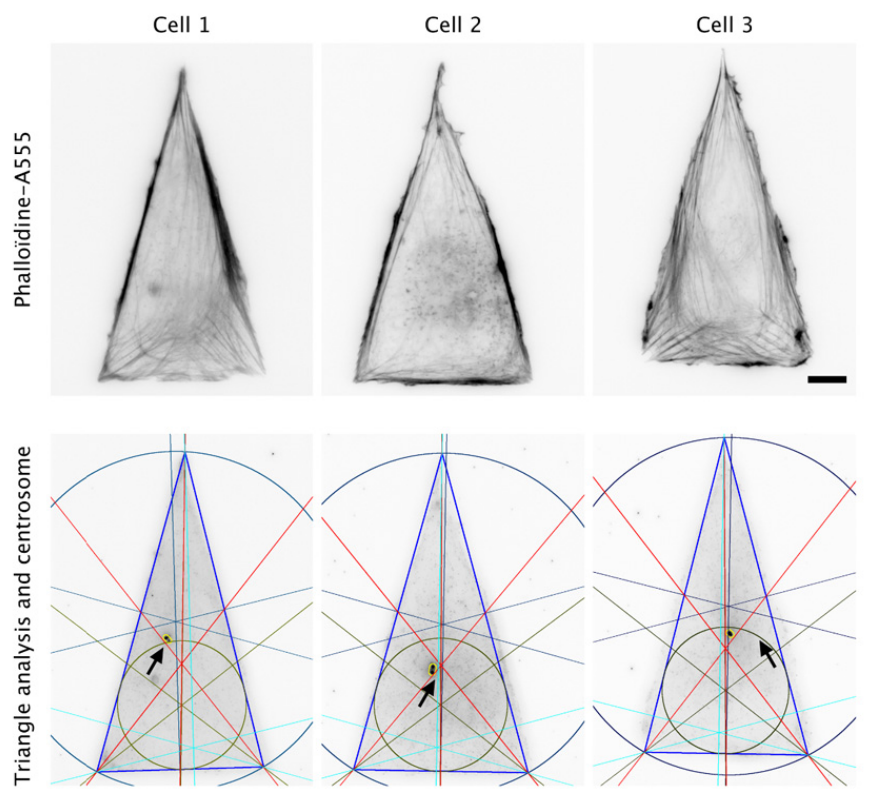

E
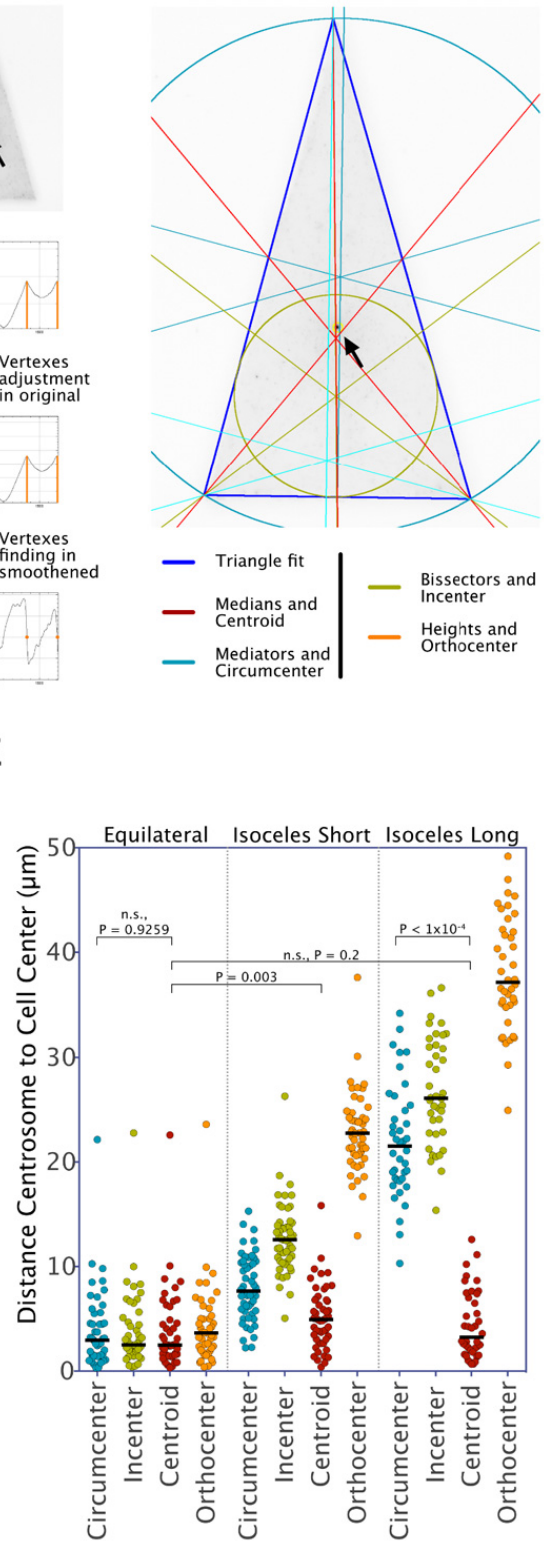

Figure S3. Triangle analysis 
A. Description of the triangle centers used for calculations of distances to the centrosome.

B. Automatic analysis overview: contour and cell centroid were determined with actin staining. The contour ROI was converted into a spline. The distance between of each vertex of the spline and the cell centroid was calculated to create the "Plot of Distances" curve. The curve was smoothened with linear regression so limit the maxima to three (as for the three triangle vertexes). Maximas were found by calculating the derivative and its sign. The corresponding vertex coordinates were used to construct the triangle fit. Geometrical definitions were used to calculate the characteristic lines and centers of each cell fitted as a triangle. All geometrical elements are plotted on the right panel in the centrosome picture. The centrosome (or centrioles) is outlined and pointed by a black arrow.

C. Several examples of the analysis are shown. Scale Bar : $10 \mu \mathrm{m}$.

D. Distance between the centrosome and the different triangle centers for three types of triangles, of $2000 \mu \mathrm{m} 2$ : equilateral, isosceles $7 / 4$ ratio (Short), isosceles 9/2 ratio (Long). 


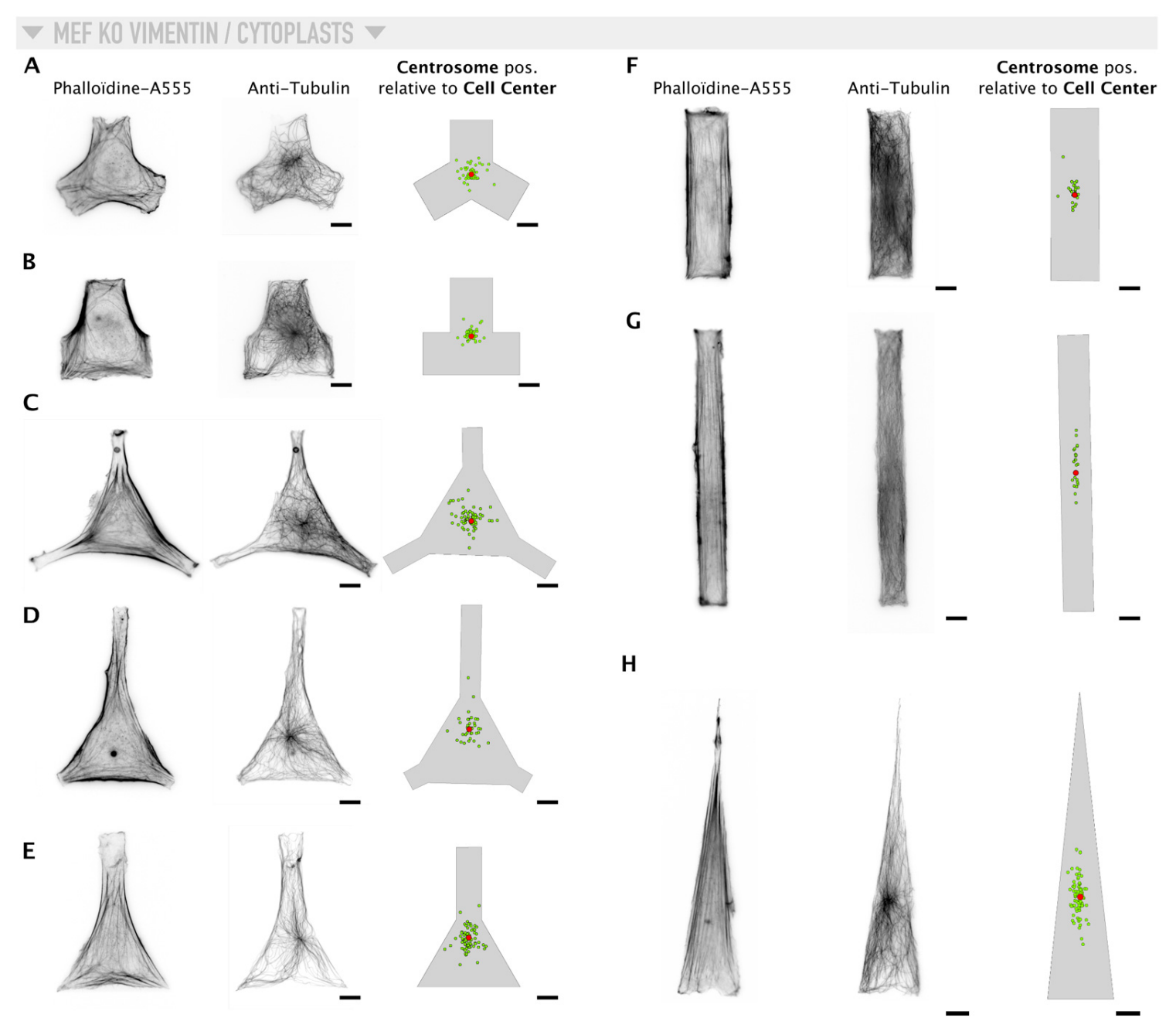

Figure S4. The centrosome positioning at the centroid of a variety of shapes.

Cytoplasts were seeded on $2000 \mu \mathrm{m} 2$ micropatterns with a variety of shapes. They were stained for microtubules and actin, and centrosome positioning was assed and plotted relative to the cell geometrical center calculated by the contour found with the actin channel. Overlapping images were taken and stitched for D,E,G and $\mathrm{H}$ before image processing. Scale bars : $10 \mu \mathrm{m}$. 


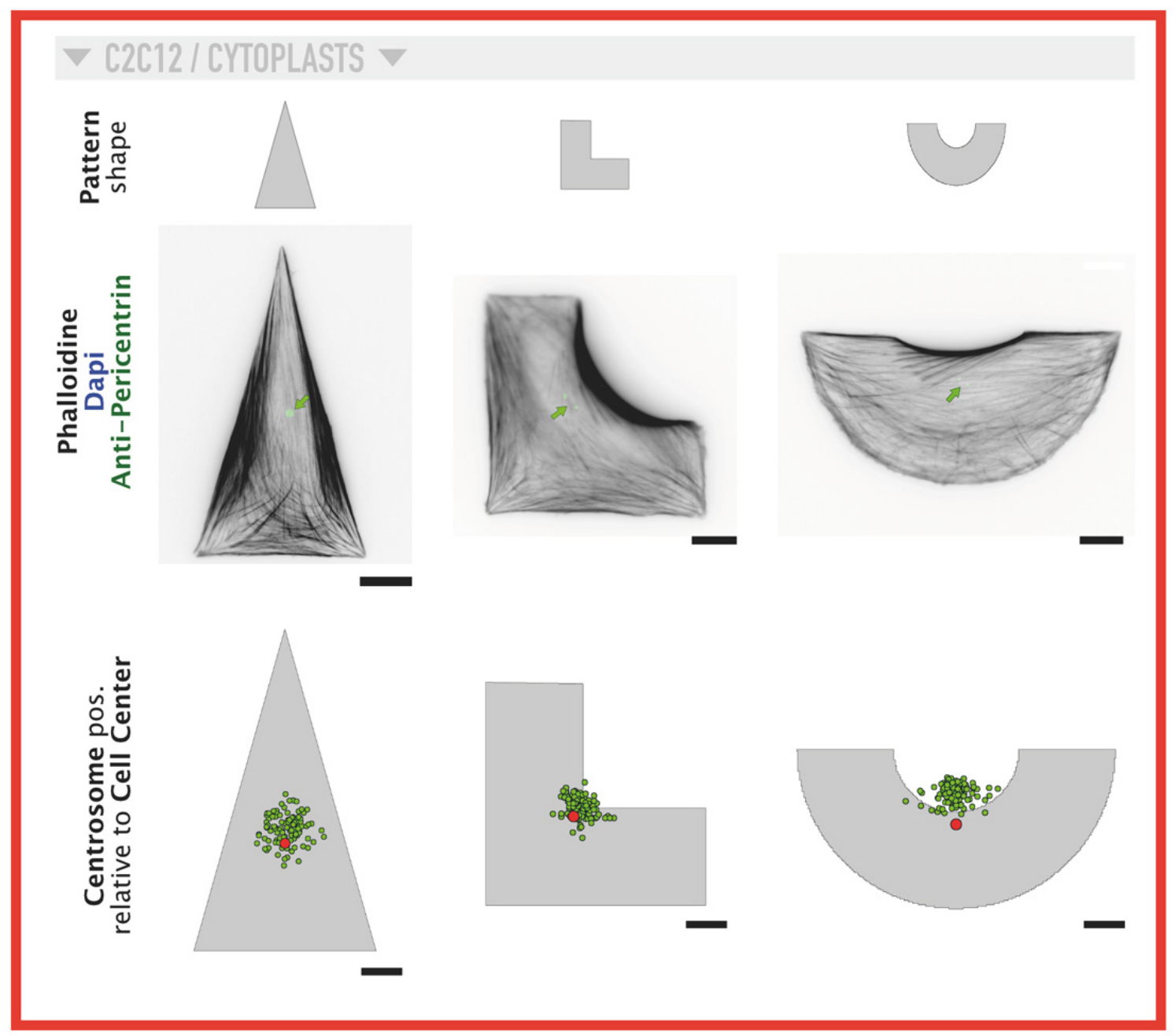

Figure S5. Centrosome is also off-centered in $\mathrm{C} 2 \mathrm{C} 12$ cytoplasts platted in anisotropic shapes.

Cytoplasts of C2C12 cells were seeded on $2000 \mu \mathrm{m} 2$ isosceles "short" triangles, L-shapes or U-Shapes. Cytoplasts were stained for actin using Phalloidine-A555 and with anti-

Pericentrine to label the centrosome. The centrosome position distribution is shifted from the cell centroid. Green arrows point at the centrosome. Scale bars : $10 \mu \mathrm{m}$. 


\section{$\checkmark$ MEF KO VIMENTIN / CYTOPLASTS $\nabla$}
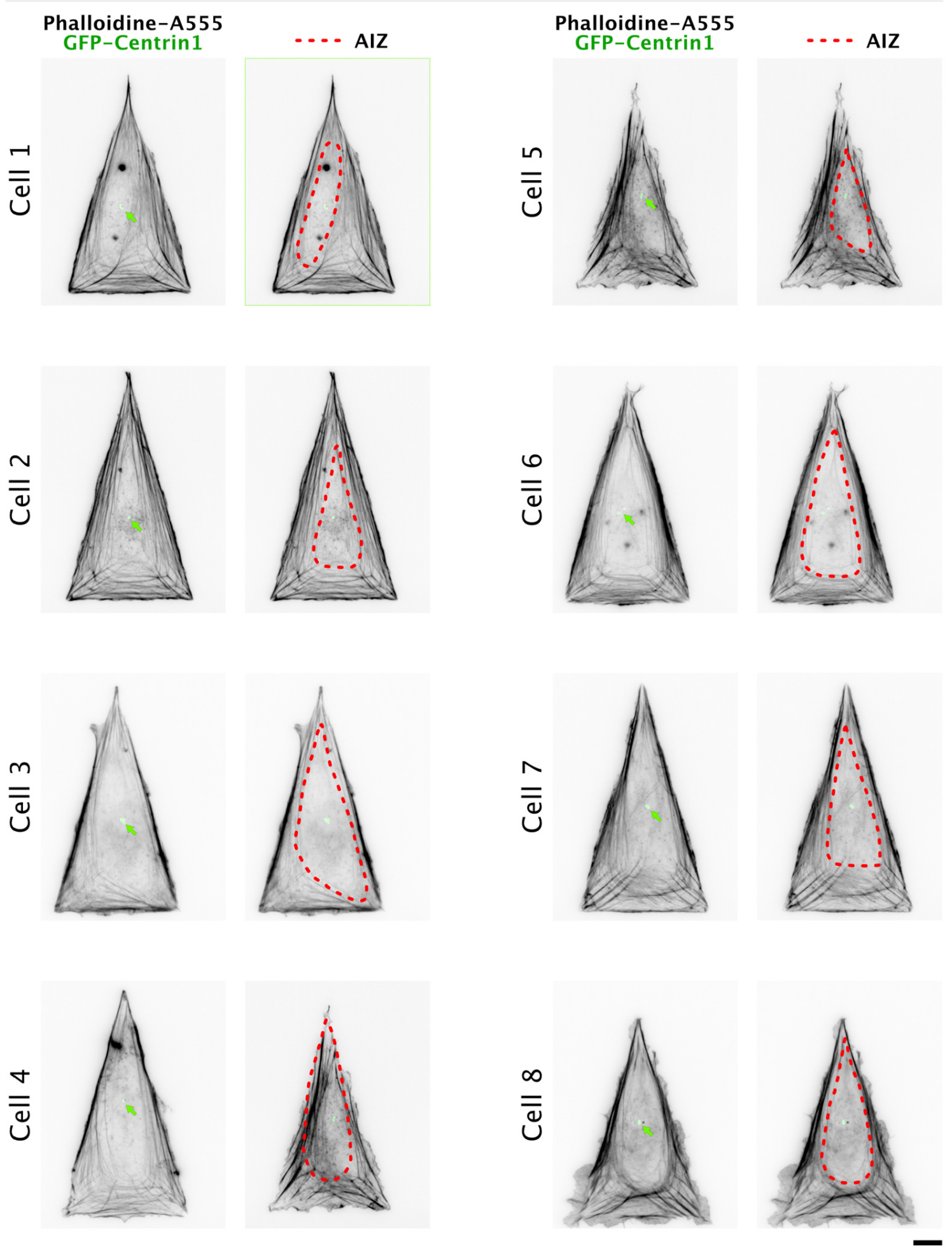

Figure S6. Centrosome positions at the center of the AIZ in triangles.

Cytoplasts were seeded on $2000 \mu \mathrm{m} 2$ isosceles "short" triangles. Cytoplasts were stained for actin using Phalloidine-A555. AIZ were determined. The figure shows several examples of the centrosome position and the AIZ in the same cell. The centrosome pointed by green arrows is centered inside the AIZ. Scale bar : $10 \mu \mathrm{m}$. 


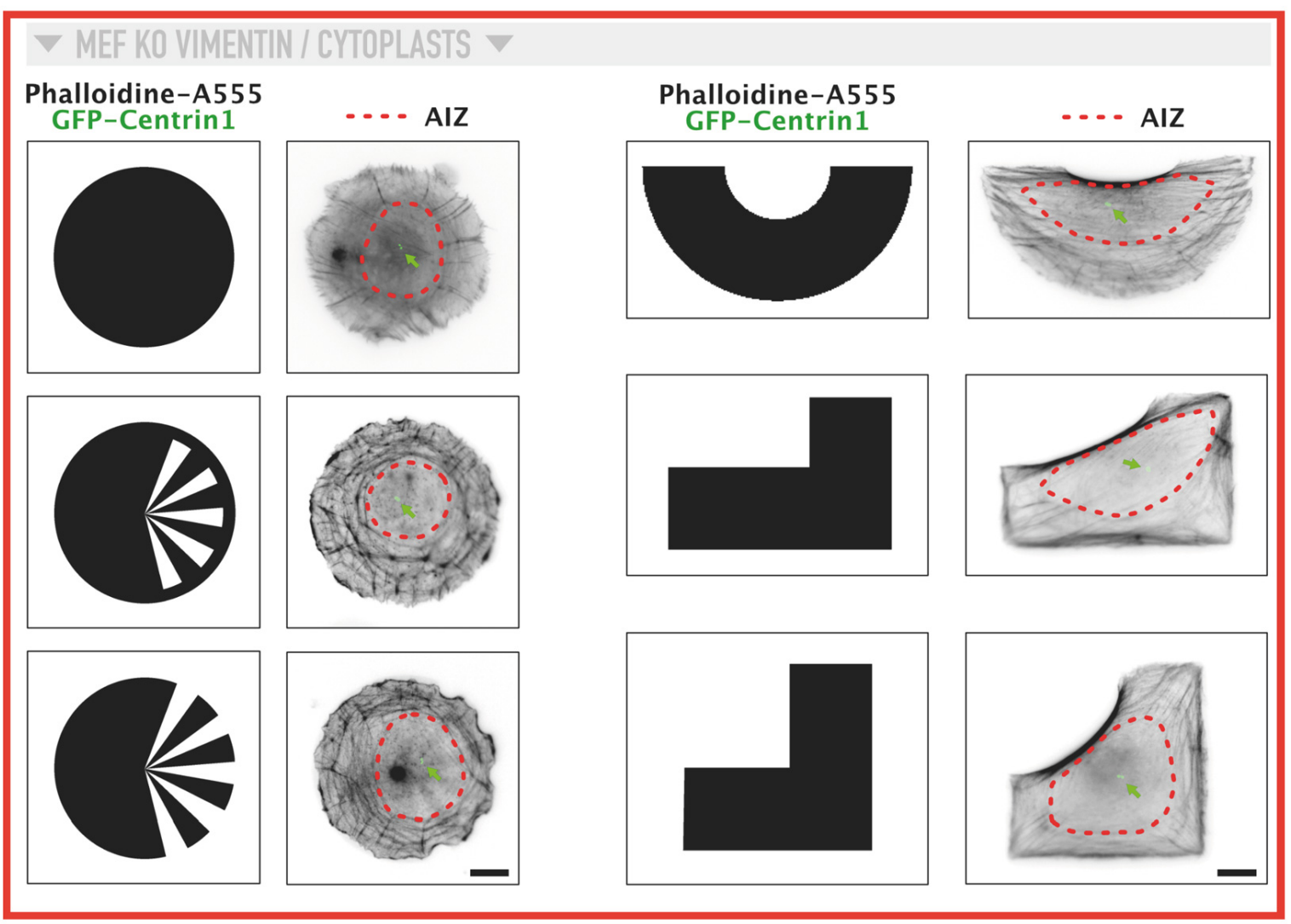

Figure S7. Centrosome positions at the center of the AIZ in different shapes.

Cytoplasts were seeded on a variety of $2000 \mu \mathrm{m} 2$ shapes presented in Figure 3. Cytoplasts were stained for actin using Phalloidine-A555. AIZ were determined. The centrosome pointed by green arrows is centered inside the AIZ. Scale bar : $10 \mu \mathrm{m}$. 


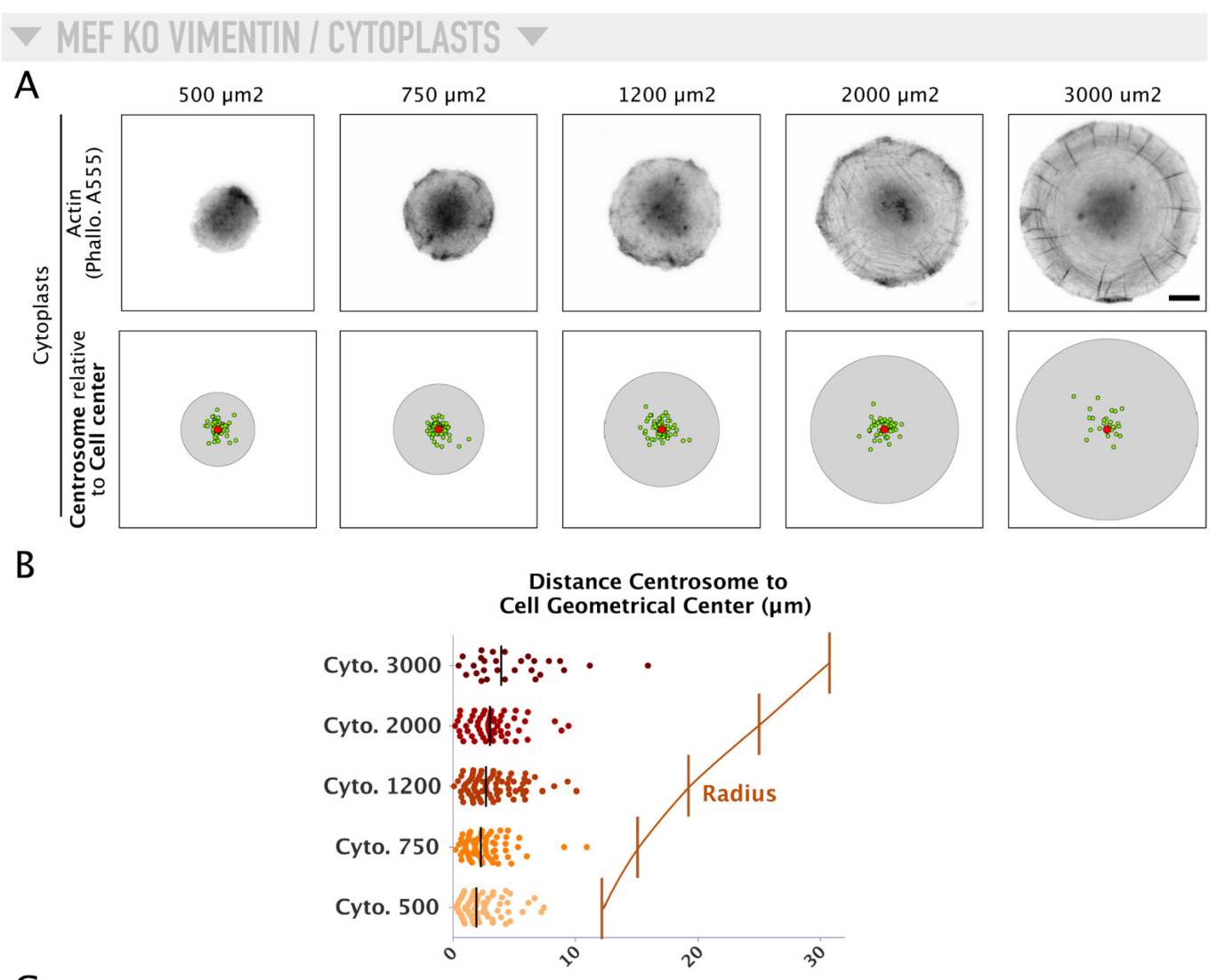

C

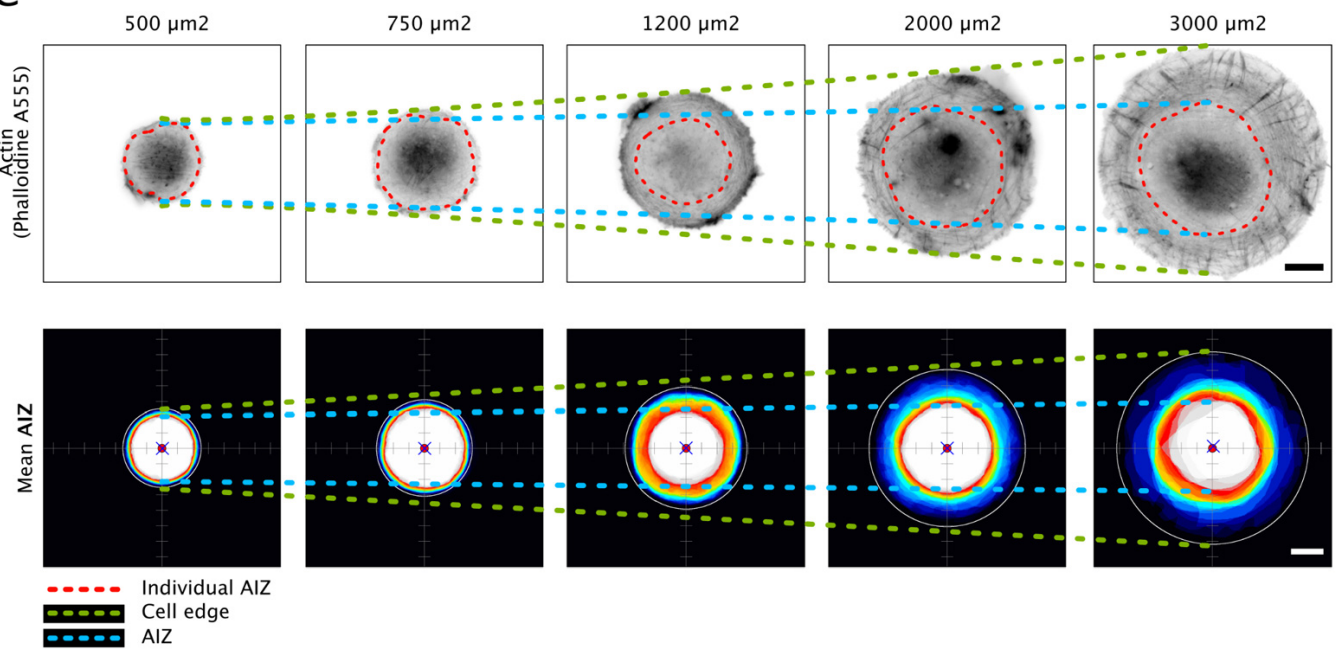

Figure S8. Centrosome positioning does not depend on cell spreading area on discshaped micropattern.

Cytoplasts were platted on disks of different sizes: 500, 750, 1200, 2000, 3000 $\mu \mathrm{m} 2$ (respective radius: 7.1, 8.7, 11.0, 14.2 and 17.4).

A. $83 \%$ of the centrosomes were found in a $5 \mu \mathrm{m}$ wide region around the geometrical center of the cell.

B. Compared to radius increase, the distance of the centrosome to the cell center remains stationary.

C. Compared to the increase of the cell diameter, the diameter of the average actin inner zone remains rather constant for all pattern sizes. Scale bars : $10 \mu \mathrm{m}$. 


\section{MEF KO VIMENTIN / CYTOPLASTS V}
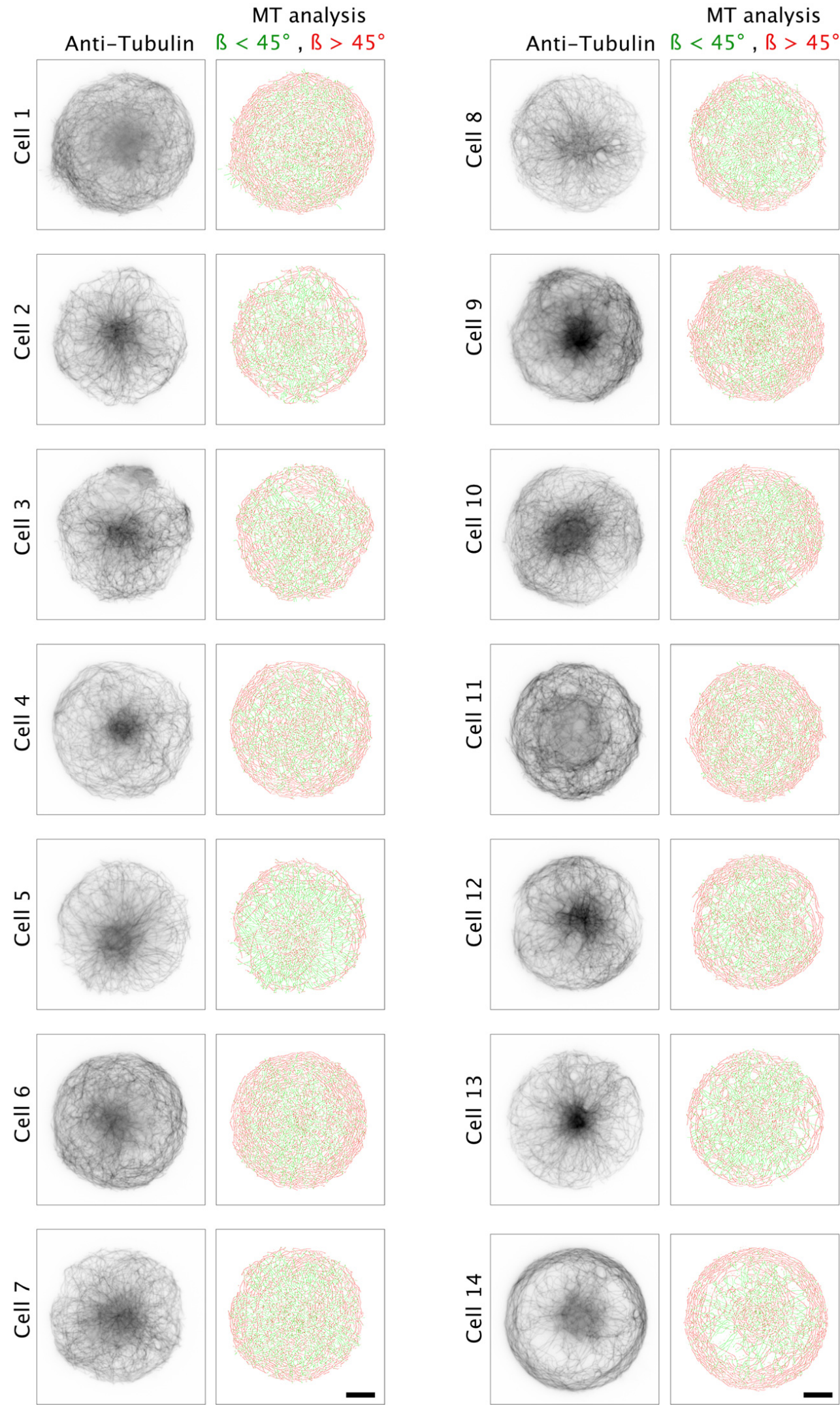
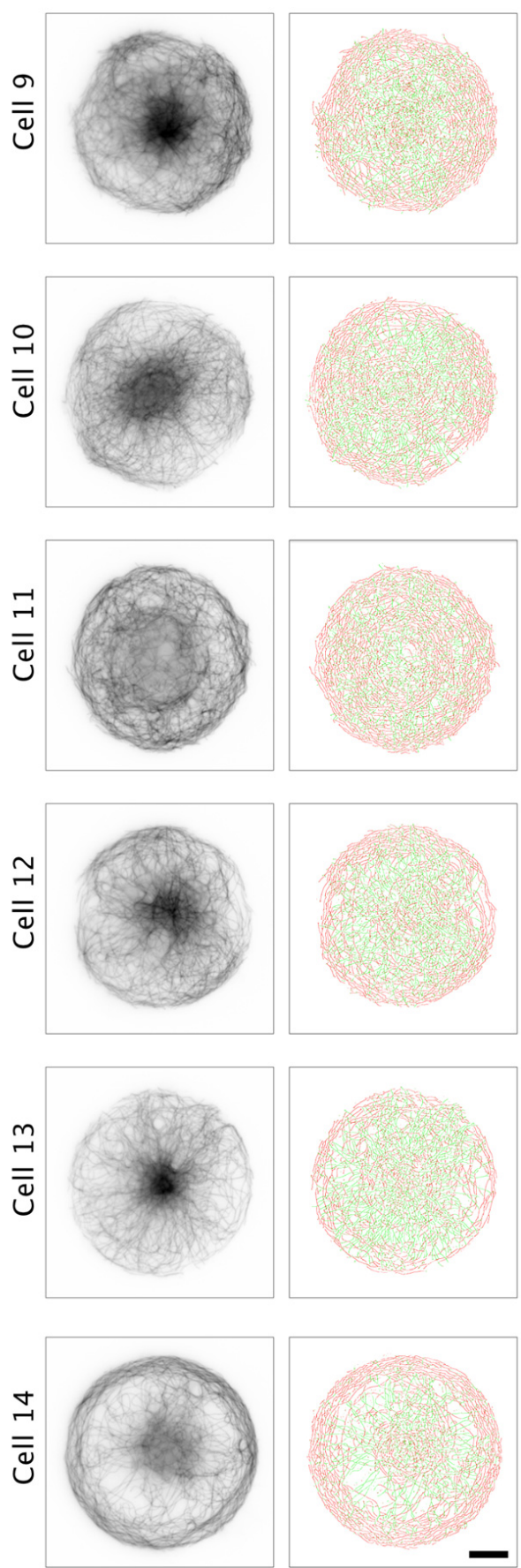

Figure S9. Analysis of microtubule orientation 
Several examples of the analysis of microtubule network orientation are presented. Cytoplasts spread on $2000 \mu \mathrm{m} 2$ disks were fixed and stained for microtubules and centrosome. Image stacks were taken and analyzed using as described in Figure 4 and in the materials and methods section, using the centrosome coordinates as the center for the calculation of the relative orientation. Best plane of microtubules in shown on the left columns and the orientation map is shown on the right columns. Scale bars : $10 \mu \mathrm{m}$. 


\section{$\checkmark$ MEF KO VIMENTIN / CYTOPLASTS *}

MT analysis
$\beta<45^{\circ}, \beta>45^{\circ}$

AIZ
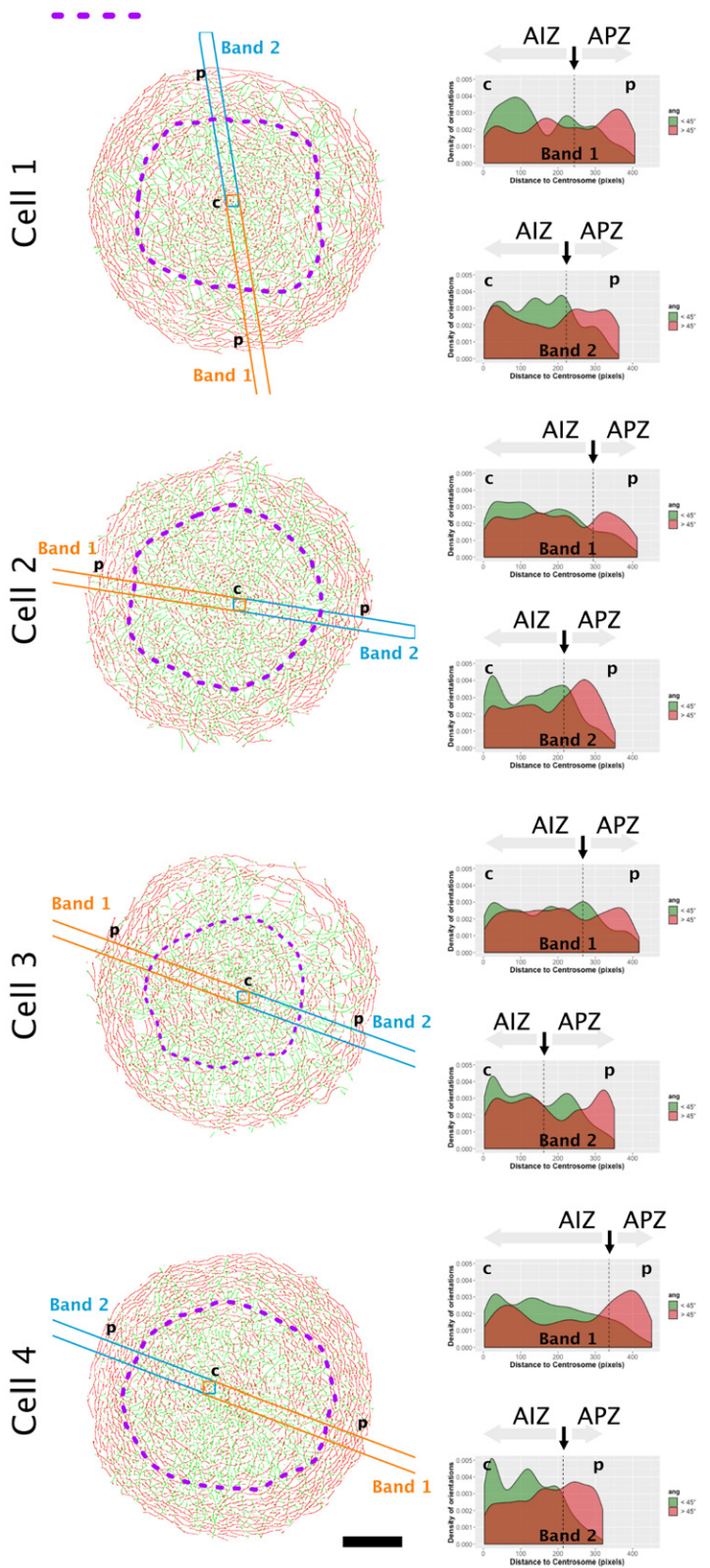

MT analysis

$B<45^{\circ}, B>45^{\circ}$

AlZ
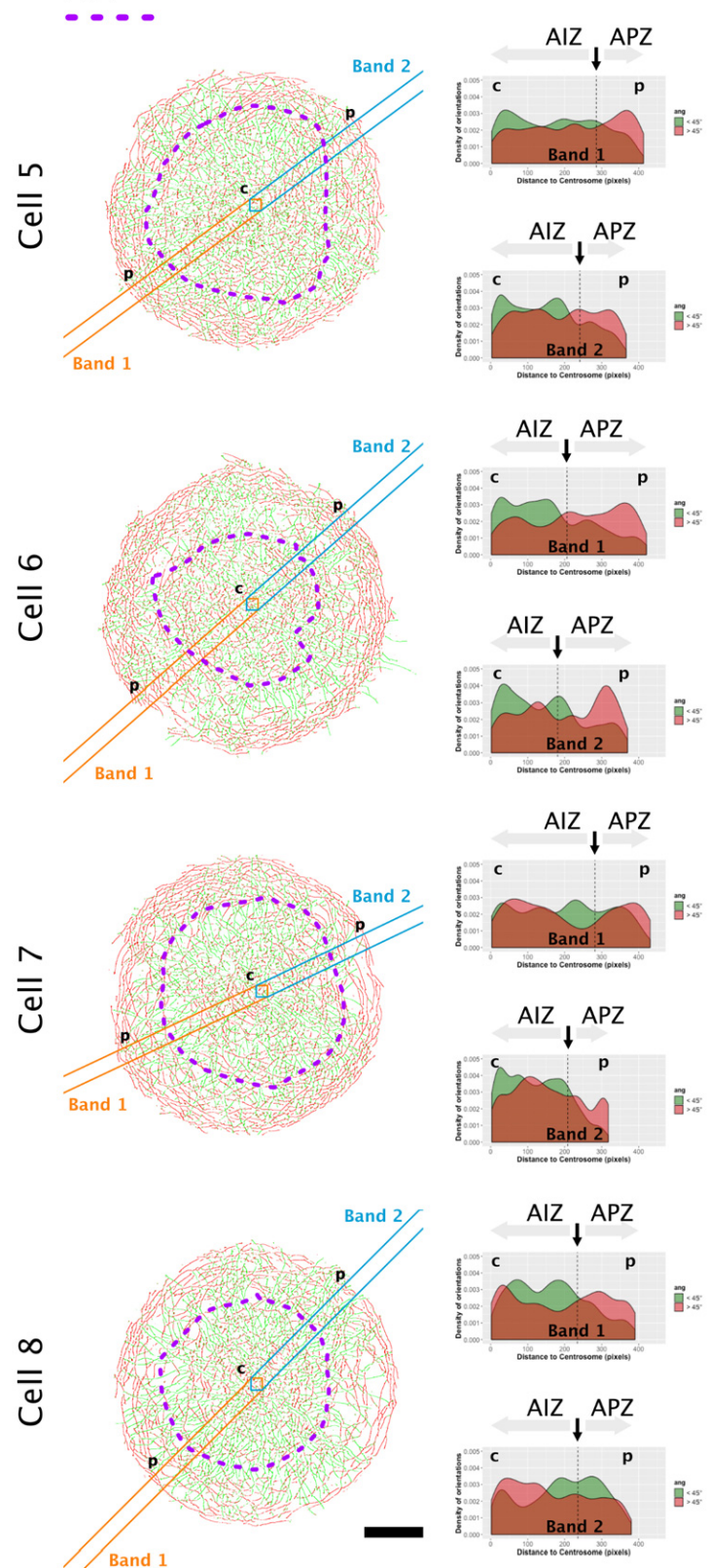

Figure S10. Analysis of microtubule orientation with respect to AIZ and APZ.

Several examples of the analysis of microtubule network orientation are presented. Cytoplasts spread on $2000 \mu \mathrm{m} 2$ disks were fixed and stained for microtubules and actin. AIZ was determined for each cell and microtubule network orientation was performed within two bands, starting from the centrosome and defined by the diameter passing by both the cell centroid and the centrosome. Radial and Tangential orientation density as a function of the distance to the centrosome were plotted using R software. Scale bars : $10 \mu \mathrm{m}$. 


\section{$\checkmark$ MEF KO VIMENTIN / CYTOPLASTS $\nabla$}
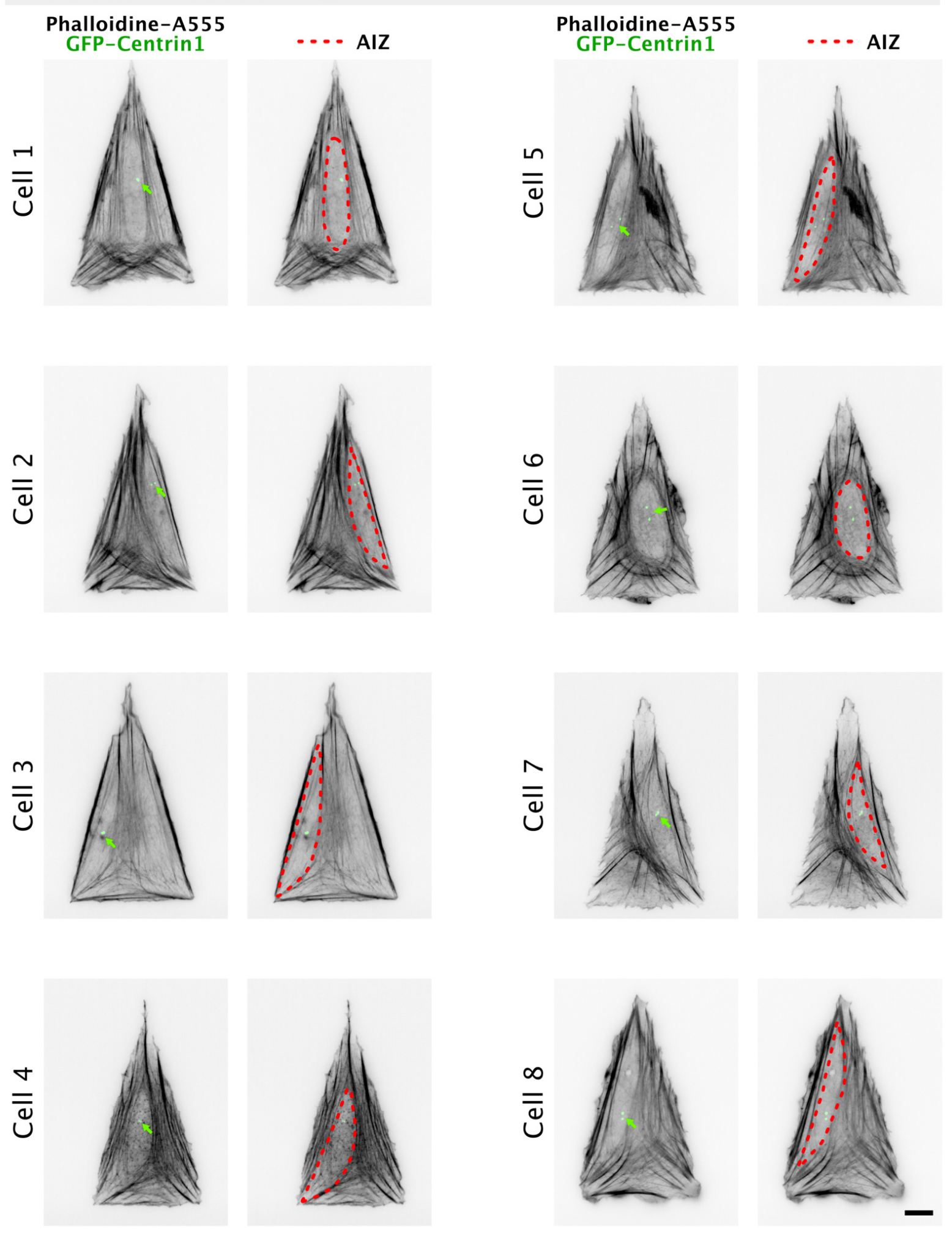

Figure S11. Microtubule disassembly shifts the AIZ and the centrosome within. 
Cytoplasts were seeded on $2000 \mu \mathrm{m} 2$ isosceles "short" triangles. They were left to spread for two hours before incubation on ice (two hours in cold room) followed Nocodazole treatment at $10 \mu \mathrm{M}$ for two hours. Cytoplasts were stained for actin using Phalloidine-A555. AIZ were determined. The figure shows several examples of the very off-centered AIZs. The centrosome, pointed by green arrows, stays inside the AIZ and follows the AIZ off-centering. Scale bar: $10 \mu \mathrm{m}$. 


\section{MEF KO VIMENTIN / CYTOPLASTS $\nabla$}

A
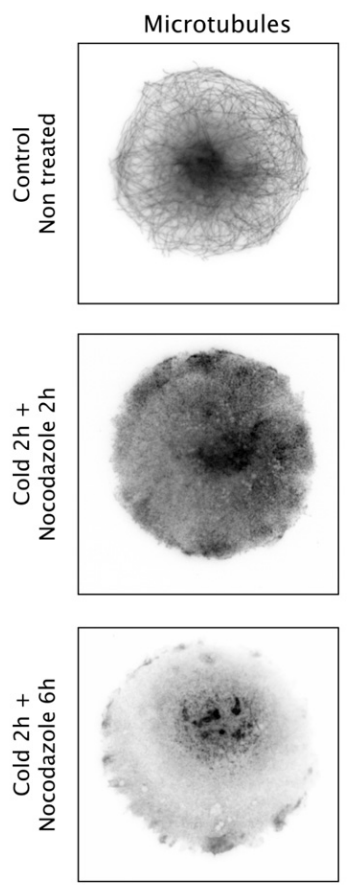

B
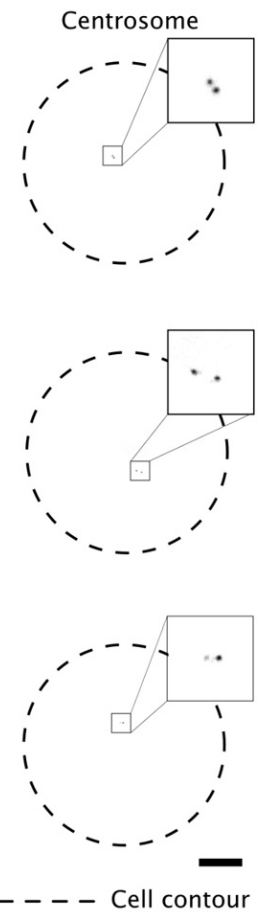
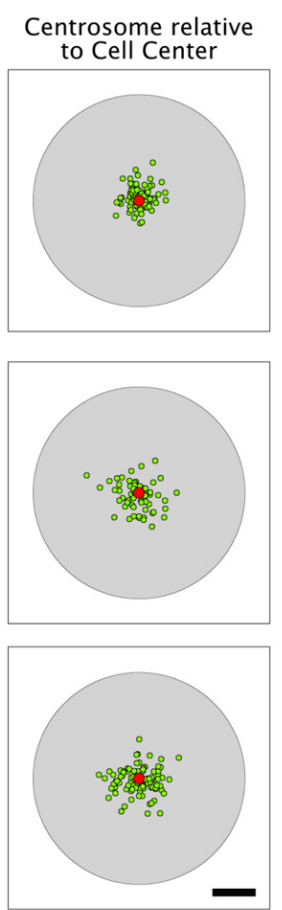

dist. Centrosome to Cell Center

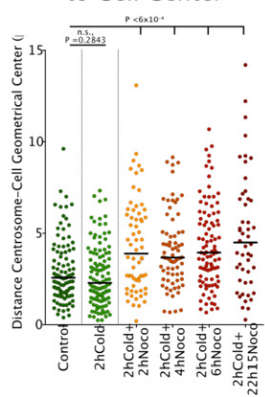

C
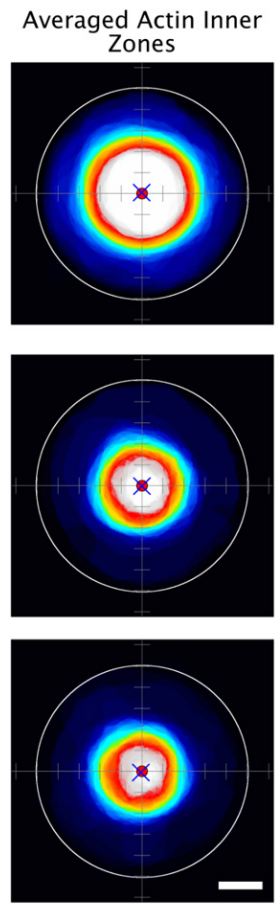

Actin Inner Area

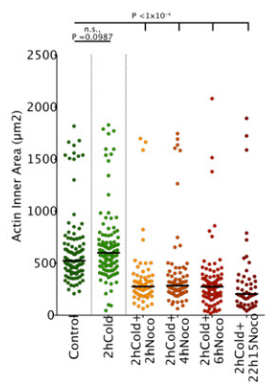

Figure S12. Centrosome dispersion upon microtubule disassembly is limited by the shrinkage of the AIZ.

Cytoplasts were platted on $2000 \mu \mathrm{m} 2$ disks (radius: 14.2).

A. Efficient removal of microtubules is achieved by incubation on ice for 2 hours followed by warming up in the presence of $10 \mu \mathrm{M}$ Nocodazole and incubation for different lapses of time. This treatment allowed to efficiently remove all microtubules (Lower left panels).

B. Plots of all centrosomes relative to the cell center and quantification of the distance between the centrosome and the cell centroid for different time points of nocodazole treatment.

C. Averages AIZs for the indicated conditions and quantification of the AIZ area for different time points of nocodazole treatment.

Red dot represents cell geometrical center or the AIC as indicated by the graph title. In C, the red dot represents the cell geometrical center and the blue cross represents the averaged relative AIC. The light-green dots are the centrosomes. Green arrows point at the centrosome. Scale bars : $10 \mu \mathrm{m}$. 


\section{MEF WT or KO VIMENTIN / CYTOPLASTS}

A

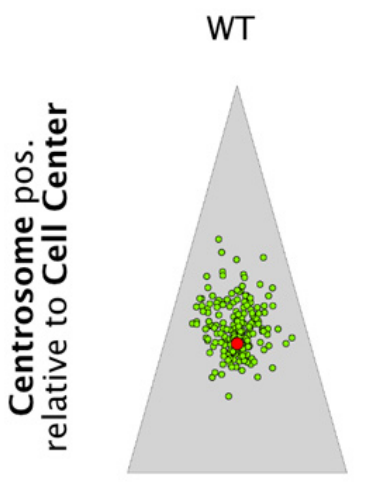

KO Vimentin
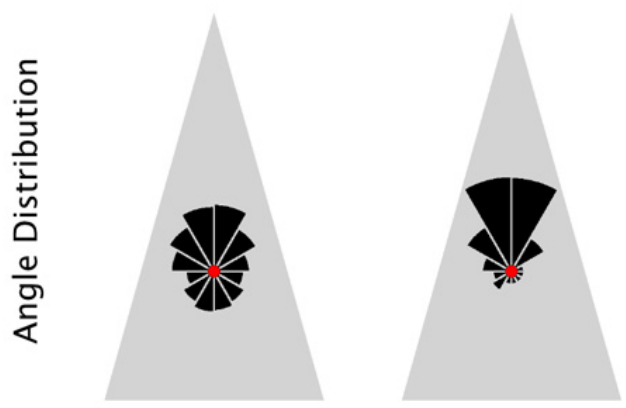

B

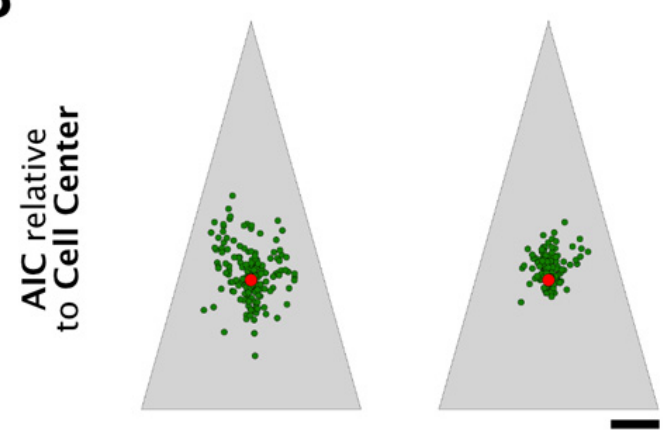

Figure S13. Centrosome and AIC positioning in WT and Vimentin-KO cells seeded in triangles.

Cytoplasts were seeded in "short" $2000 \mu \mathrm{m} 2$ isosceles triangles and stained for centrosome and actin.

A. The centrosome position in both cell lines present the same asymmetry towards the cell apex although it is slightly more dispersed in WT derived cytoplasts.

B. A less centered AIZ seems to be responsible for this mild difference. This off-centering could be due increased contractility or the presence of Vimentin cages.

The red dots represent the cell centroid. Scale bar : $10 \mu \mathrm{m}$. 


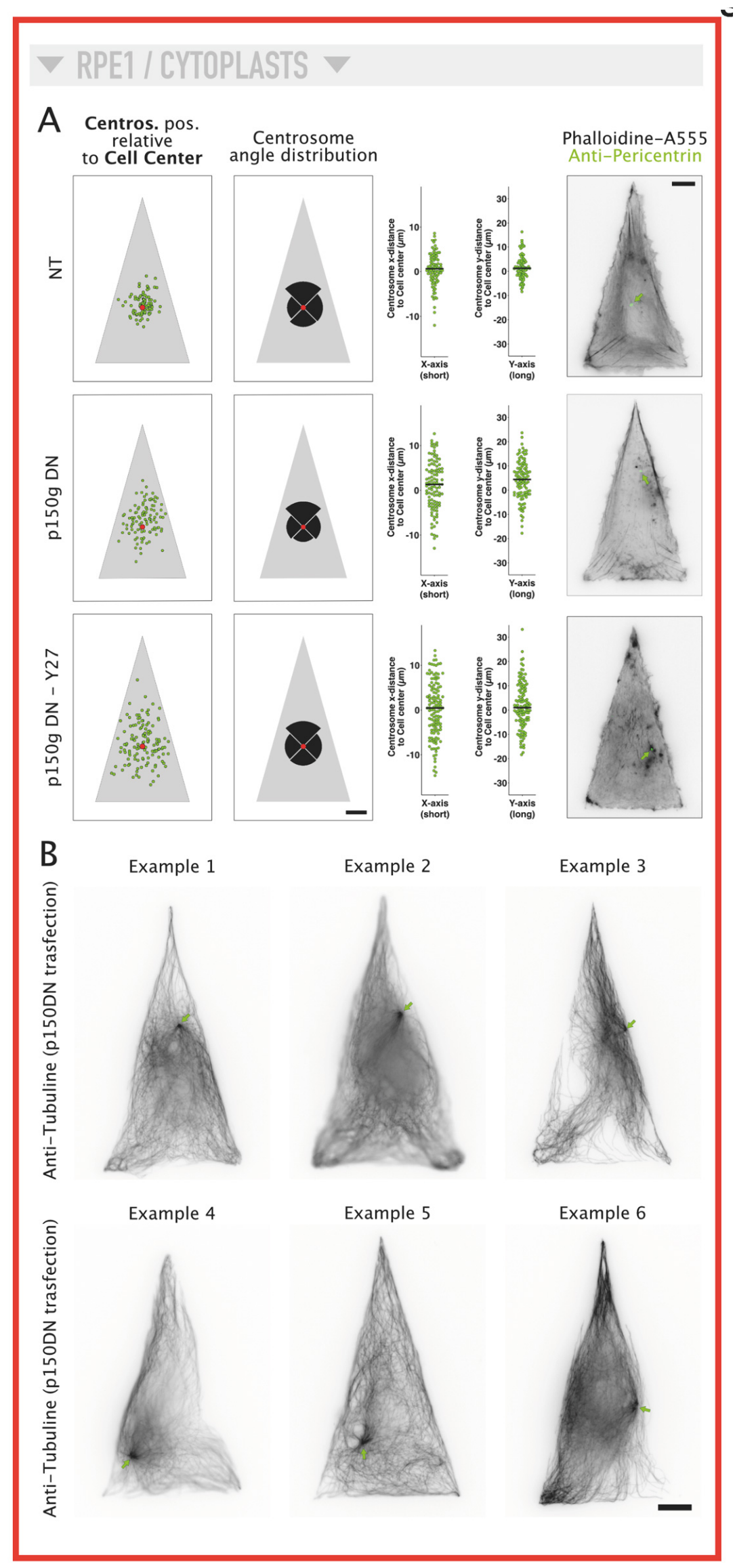

Figure S14. Role of dyneins in centrosome positioning in the AIZ using RPE1 cytoplasts 
Cytoplasts of RPE1 cells electroporated with p150-DN 48 hours before enucleation and positive cells were sorted by flow cytometry $24 \mathrm{~h}$ before enucleation. Cytoplasts were plated on $2000 \mu \mathrm{m} 2$ Isosceles "Short" for 2 hours and treated when indicated with Y27632 at $200 \mu \mathrm{M}$ for 2 extra hours.

A. The centrosome is dispersed in all conditions but with a small bias towards the cell apex as shown by the distances along the y-axis plots in the third column. Examples of centrosomes centered in control conditions and off-centered in $\mathrm{p} 150 \mathrm{gDN}$ conditions are shown.

B. Examples of centrosome off-centering an microtubule network shapes in p150g-DN transfected cell.

Light green arrows point at the centrosomes. Scale bars: $10 \mu \mathrm{m}$. 


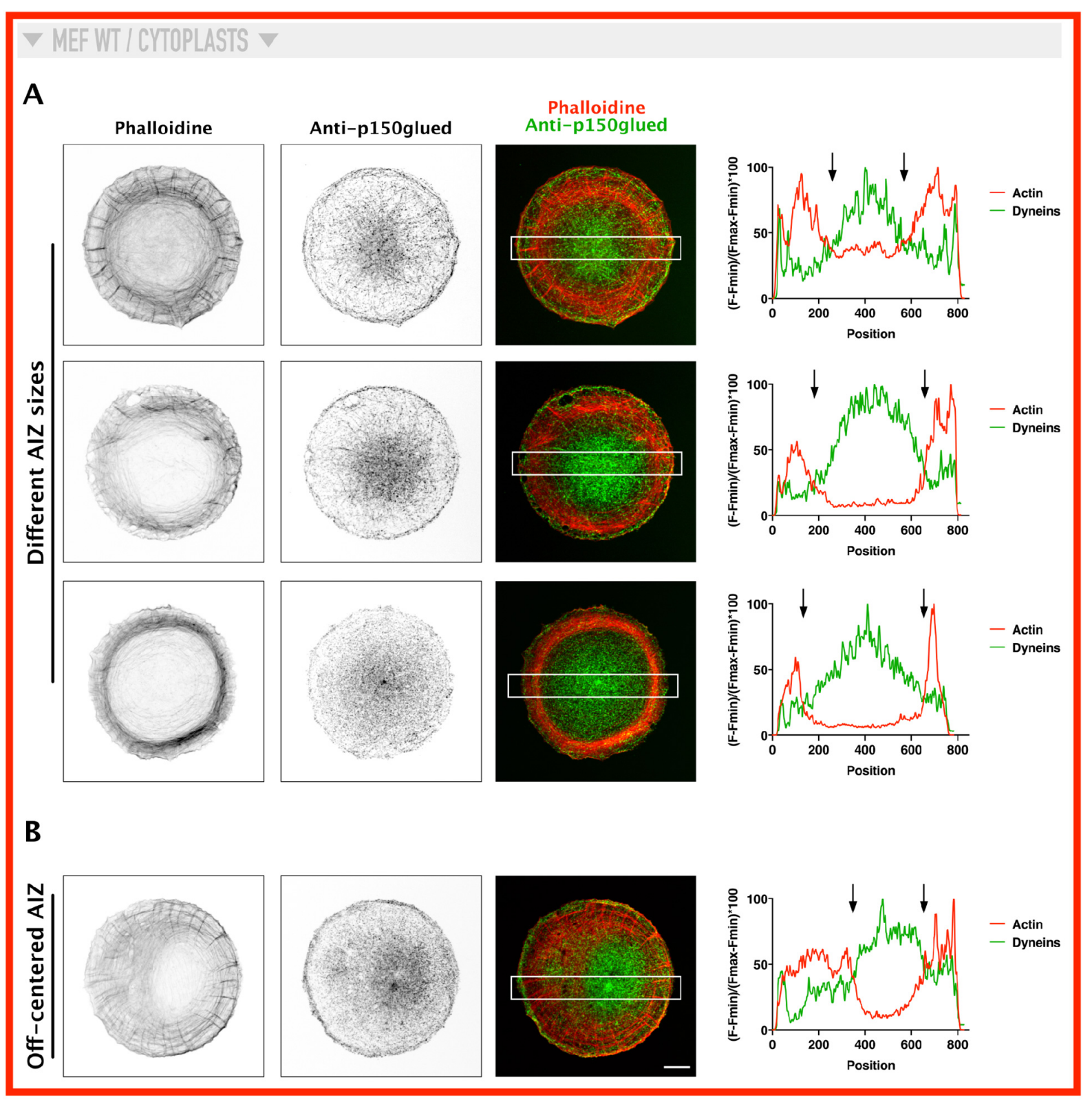

Figure S14. Spatial distributions of dyneins in response to AIZ geometry

A,B Cytoplasts were plated on $2000 \mu \mathrm{m} 2$, fixed and immunolabelled for actin and p150Glued. Linescans show the intensity of the two signals along the rectangle indicated in the overlay. Various examples are displayed showing the enlargment of the distribution of p150Glued when the AIZ is wider (A) and the off-centering of the distribution of p150Glued when the $\mathrm{AIZ}$ is asymmetric (B). Arrows show the boundaries of the AIZ. 